\title{
Coherent Versus Non-Coherent Decode-and-Forward Relaying Aided Cooperative Space-Time Shift Keying
}

\author{
Shinya Sugiura, Member, IEEE, Sheng Chen, Fellow, IEEE, Harald Haas, Member, IEEE, \\ Peter M. Grant, Fellow, IEEE, and Lajos Hanzo, Fellow, IEEE
}

\begin{abstract}
Motivated by the recent concept of Space-Time Shift Keying (STSK), we propose a novel cooperative STSK family, which is capable of achieving a flexible rate-diversity tradeoff, in the context of cooperative space-time transmissions. More specifically, we first propose a Coherent cooperative STSK (CSTSK) scheme, where each Relay Node (RN) activates Decodeand-Forward (DF) transmissions, depending on the success or failure of Cyclic Redundancy Checking (CRC). We invoke a bitto-STSK mapping rule, where according to the input bits, one of the $Q$ pre-assigned dispersion vectors is activated to implicitly convey $\log _{2} Q$ bits, which are transmitted in combination with the classic $\log _{2} \mathcal{L}$-bit modulated symbol. Additionally, we introduce a beneficial dispersion vector design, which enables us to dispense with symbol-level Inter-Relay Synchronization (IRS). Furthermore, the Destination Node (DN) is capable of jointly detecting the signals received from the source-destination and relaydestination links, using a low-complexity single-stream-based Maximum Likelihood (ML) detector, which is an explicit benefit of our Inter-Element Interference (IEI)-free system model. More importantly, as a benefit of its design flexibility, our cooperative CSTSK arrangement enables us to adapt the number of the RNs, the transmission rate as well as the achievable diversity order. Moreover, we also propose a Differentially-encoded cooperative STSK (DSTSK) arrangement, which dispenses with CSI estimation at any of the nodes, while retaining the fundamental benefits of the cooperative CSTSK scheme.
\end{abstract}

Index Terms-Asynchronous cooperation, cooperative diversity, diversity versus multiplexing tradeoff, space-time coding, space-time shift keying, spatial modulation.

\section{INTRODUCTION}

I $\mathrm{N}$ recent years, cooperative Space-Time Codes (STCs) [1][3] have been extensively investigated due to their potential to achieve a high transmit diversity gain, where a collection of distributed nodes act as a Virtual Antenna Array (VAA), which are positioned sufficiently far apart for experiencing uncorrelated Multiple-Input Multiple-Output (MIMO) channels.

Paper approved by D. I. Kim, the Editor for Spread Spectrum Transmission and Access of the IEEE Communications Society. Manuscript received September 1, 2010; revised December 15, 2010 and February 18, 2011.

The financial support of the EU under the auspices of the Optimix project and of the EPSRC UK is gratefully acknowledged.

S. Sugiura is with Toyota Central R\&D Laboratories, Inc., Aichi, 480-1192, Japan (e-mail: sugiura@mosk.tytlabs.co.jp).

S. Chen and L. Hanzo are with the School of Electronics and Computer Science, University of Southampton, Southampton, SO17 1BJ, U.K. (e-mail: $\{$ sqc, lh\}@ecs.soton.ac.uk).

H. Haas and P. M. Grant are with the Institute for Digital Communications (IDCOM), The University of Edinburgh, Edinburgh, U.K. (e-mail: \{h.haas, Peter.Grant\}@ed.ac.uk).

Digital Object Identifier 10.1109/TCOMM.2011.042111.100536
Furthermore, this VAA arrangement enables us to avoid the employment of multiple RF chains at each node.

On the other hand, attaining a high cooperative spacetime diversity gain in a practical relay-aided network imposes further challenges. Firstly, most of the previously-proposed cooperative STCs assumed perfect Inter-Relay Synchronization (IRS), although it is a challenging task to acquire symbollevel timing synchronization between the distributed Relay Nodes (RNs). However, as noted in [4], the asynchronous transmissions of the RNs may destroy the STC's orthogonality, leading to a severe performance degradation. For the sake of effectively combating this IRS problem, a number of asynchronous cooperative STCs were proposed in [5]-[7]. Another challenge is the mitigation of Channel State Information (CSI) estimation errors for the Source-Destination (SD), the Source-Relay (SR) and/or of the Relay-Destination (RD) links at the RNs or the Destination Node (DN). Practically, the rapidly changing topology of vehicles travelling at high speeds makes it challenging to acquire accurate CSI, which results in a severe degradation of the achievable performance. Since each of the MIMO subchannels has to be sampled above the Doppler frequency, at high speeds an increased pilot overhead is added for the sake of accurately estimating each MIMO channel component, which also gives rise to a substantial increase of the complexity. While the majority of the cooperative STC studies assumed availability of perfect CSI, a number of cooperative Differential STCs (DSTCs) were proposed in [8], [9] in order to achieve reliable symbol detection without any CSI.

Recently, the sophisticated concept of Spatial Modulation (SM) [10]-[12] was proposed for co-located MIMO elements, where only one of the $M$ available transmit antennas is activated within each symbol interval, which serves as an additional implicit means of conveying information, over and above the conventional symbol constellation. As a benefit, in contrast to Vertical Bell Laboratories Layered (V-BLAST), the resultant system model does not suffer from any Inter-Element Interference (IEI). Therefore, also in contrast to V-BLAST, low-complexity single-antenna-based Maximum Likelihood (ML) detection can be utilized at the receiver instead of the joint detection of multiple streams. More specifically, it was found in [10]-[12] that SM has the potential of outperforming other MIMO arrangements, such as V-BLAST and Alamouti's STBC schemes. Considering that the SM scheme 
can be operated without perfect Inter-Antenna Synchronization (IAS), our proposition is that this arrangement may also be suitable for cooperative communication scenarios, although no cooperative SM scheme has been presented in the open literature. However, since SM was not designed for providing transmit diversity gain, increasing the number of RNs would not increase the cooperative diversity order.

More recently, in [13] the novel concept of Space-Time Shift Keying (STSK) was invented, where space-time codewords are generated by activating one out of $Q$ space-time dispersion matrices, rather than one out of $M$ antenna elements as in the SM scheme [10]-[12]. The STSK scheme is capable of achieving a flexible tradeoff between the attainable diversity and throughput, hence outperforming other MIMO arrangements, such as OSTBCs, BLAST and SM schemes. ${ }^{1}$ Furthermore, since no Inter-Channel Interference (ICI) is imposed by the STSK receiver, low-complexity single-streambased ML detection may be invoked. Furthermore, in addition to the above-mentioned Coherent STSK (CSTSK), the corresponding Differentially-encoded STSK (DSTSK) arrangement was also proposed in [13], where no CSI estimation was required at the receiver, at the cost of typical $3-\mathrm{dB}$ penalty in comparison to the CSTSK scheme, which is a consequence of the error-doubling property of differential decoding.

Motivated by the recent concept of STSK [13], in this paper we develop two novel cooperative STC architectures, i.e. the cooperative CSTSK and DSTSK schemes. The novel contributions of this paper are as follows.

- We first propose a cooperative CSTSK scheme, which acts as a unified shift keying architecture designed for achieving a useful cooperative diversity. Our scheme is capable of activating an arbitrary number of RNs, as well as of appropriately adjusting both the transmission rate and the diversity gain. More specifically, each RN is a constituent part of our distributed Decode-and-Forward $(D F)$ scheme, where one out of $Q$ pre-assigned dispersion vectors is activated and transmitted within each block duration for transmitting a PSK/QAM symbol. Moreover, each relay participates in the cooperative regime in a distributed manner, namely without negotiating with the SN or RNs, hence achieves a substantial reduction of the overhead compared to coordinated DF schemes.

- Furthermore, by exploiting the fact that no IEI is imposed at the DN in our cooperative CSTSK scheme, we derive a single-stream-based low-complexity ML detector, whose complexity is comparable to that of Orthogonal SpaceTime Block Coding (OSTBC) schemes [15], [16].

- We also demonstrate that by appropriately designing the $Q$ pre-assigned dispersion vectors, the RNs of the cooperative CSTSK scheme is capable of dispensing with the requirement of perfectly synchronizing their transmissions with other RNs, which leads to a high robustness against IRS errors limited to a fraction of the symbol duration.

- Moreover, inspired by the DSTSK scheme designed for the co-located MIMO arrangement [13], we conceive a

\footnotetext{
${ }^{1}$ To expound a little further, in [14] it was demonstrated that the generalized STSK scheme subsumes many other MIMO arrangements, such as OSTBCs, BLAST, LDCs and SM schemes.
}

cooperative DSTSK scheme, where the nodes dispense with CSI estimation, while retaining the fundamental benefits of the cooperative CSTSK scheme.

The remainder of this paper is organized as follows. In Section II, the system model of our cooperative CSTSK scheme and its detection algorithm employed at the destination receiver are presented. Section III introduces the differentially-encoded counterpart of the cooperative CSTSK scheme. Section IV provides our performance results, while our conclusions are presented in Section V.

\section{System Model of CoOperative CSTSK Scheme}

This section describes our cooperative CSTSK scheme. As seen in Fig. 1, we consider a two-phase relay network, which is constituted by a single Source Node ( $\mathrm{SN}), M$ RNs and a DN, each having a single antenna element. We note that the proposed scheme can be readily extended to the multipleantenna-element assisted DN scenario. Here, it is assumed that a Time Division Multiple Access (TDMA) protocol is used and that the cooperating relays attempt to loosely-synchronize their timings under the BS's control. ${ }^{23}$ We also assume that each node is operated in a half-duplex mode, either receiving or transmitting in a given time slot. Additionally, for the sake of enabling the CRC at the RNs, frame-based rather than symbol-based transmissions are carried out, although this leads to an increased detection delay.

\section{A. Source Model}

During the broadcast phase of Fig. 1, the SN transmits its information to the $M \mathrm{RNs}$ as well as to the DN. As noted in [3], the SN first attaches Cyclic Redundancy Check (CRC) bits to its information bits in order for the RNs to be able to detect the potential decoding errors and hence to avoid the propagation of DF errors to the DN. Then the CRC-encoded bits are mapped to the $\mathcal{L}^{\prime}$-constellation point PSK/QAM scheme in order to generate the symbols $\boldsymbol{S}_{\mathrm{s}}(i)=\left[s_{1}(i), \cdots, s_{b}(i)\right]^{T} \in \mathcal{C}^{b \times 1}$, where $i$ represents the block index and $b \log _{2} \mathcal{L}^{\prime}$ bits are transmitted in each block. Let us assume that the SN transmits the symbols $\boldsymbol{S}_{\mathrm{s}}(i)$ over $b$ time slots, and the corresponding signals received at the $m$ th $\mathrm{RN}$ as well as at the $\mathrm{DN}$ are given by

$$
\boldsymbol{Y}_{\mathrm{sr}}^{(m)}(i)=h_{\mathrm{sr}}^{(m)}(i) \boldsymbol{S}_{\mathrm{s}}(i)+\boldsymbol{N}_{\mathrm{r}}^{(m)}(i) \quad\left(i=1, \cdots, L_{\mathrm{f}}\right)
$$

and

$$
\boldsymbol{Y}_{\mathrm{sd}}(i)=h_{\mathrm{sd}}(i) \boldsymbol{S}_{\mathrm{s}}(i)+\boldsymbol{N}_{\mathrm{d}}(i) \quad\left(i=1, \cdots, L_{\mathrm{f}}\right),
$$

respectively, where the channel coefficients $h_{\mathrm{sr}}^{(m)}$ and $h_{\mathrm{sd}}(i)$ obey the complex-valued Gaussian distributions of $\mathcal{C N}\left(0, \sigma_{\text {sd }}^{2}\right)$ and of $\mathcal{C N}\left(0, \sigma_{\mathrm{sr}}^{2}\right)$, while each component of the noise vectors $\boldsymbol{N}_{\mathrm{r}}^{(m)}(i)$ and $\boldsymbol{N}_{\mathrm{d}}(i)$ is a complex-valued Gaussian variable of $\mathcal{C N}\left(0, N_{0}\right)$. Furthermore, $N_{0}$ represents the noise variance and $L_{\mathrm{f}}$ denotes the number of $b \log _{2} \mathcal{L}^{\prime}$-bit signal blocks, which are successively transmitted in each single transmission frame during the broadcast phase.

\footnotetext{
${ }^{2}$ For the sake of simple relay activation and the coarse timing control, we assumed the presence of a system controller at the BS of cellular networks.

${ }^{3}$ The synchronization accuracy achieved by the Medium Access Control (MAC) layer is typically lower than the symbol-synchronized simultaneous transmissions of the relays.
} 


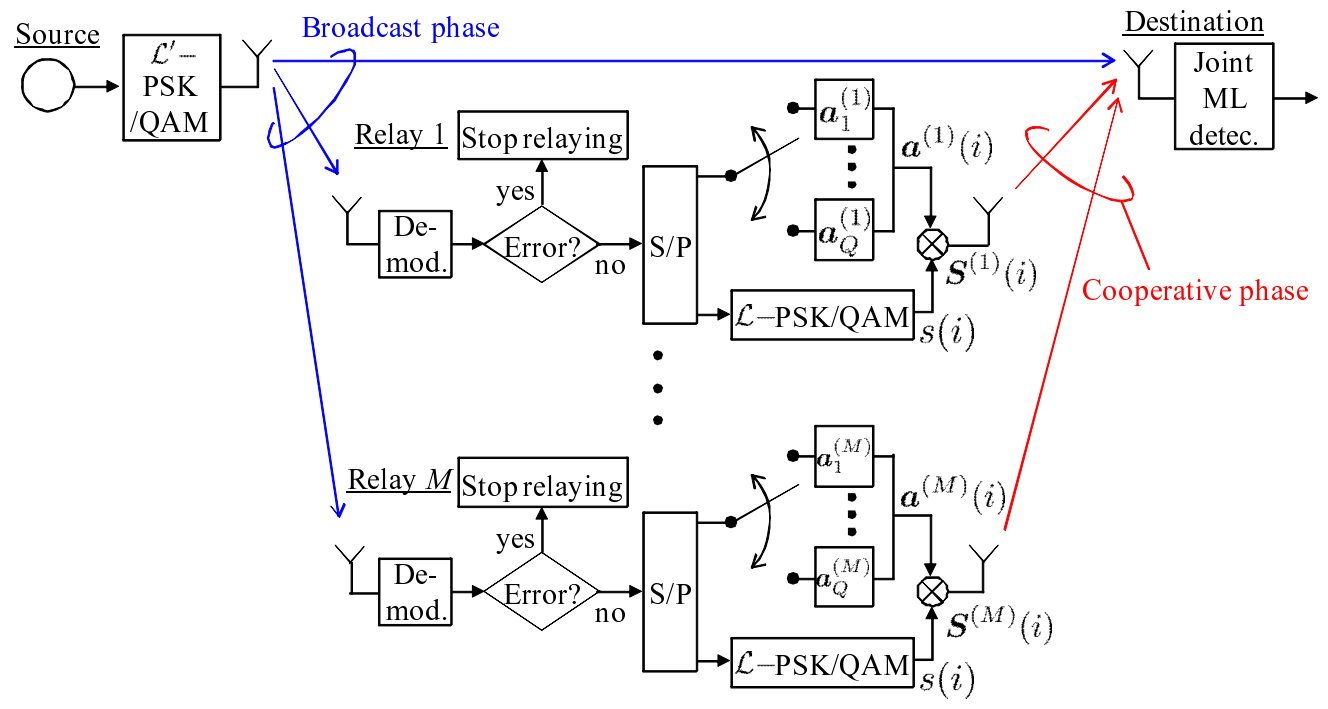

Fig. 1. Schematic of our cooperative CSTSK scheme assisted by selective DF relaying.

\section{B. Relay Model}

Let us now describe the cooperative phase of Fig. 1, where the $M$ RNs employ CRC-activated DF transmission with the aid of our cooperative CSTSK scheme. Let us consider the $m$ th $\mathrm{RN}$ to be the node of interest, which first decodes the received signals $\boldsymbol{Y}_{\mathrm{sr}}^{(m)}(i)$ of (1). If any decoding error is identified by the $\mathrm{CRC}$, the $\mathrm{RN}$ refrains from relaying the signals to the DN without requiring any negotiation with the other nodes. By contrast, if there are no decoding errors, the $m$ th relay re-encodes the decoded bits and transmits them using the D-STSK scheme as follows. The $b \log _{2} \mathcal{L}^{\prime}$ decoded bits per $b$-slot block are Serial-to-Parallel (S/P) converted to $\log _{2} Q$ and $\log _{2} \mathcal{L}$ bits, assuming the relationship of $b \log _{2} \mathcal{L}^{\prime}=\log _{2}(Q \cdot \mathcal{L})$. Here, we will represent each of the corresponding $\mathrm{S} / \mathrm{P}$ converted bits as $(q, l)$ in decimal representation. Then, as shown in Fig. 1, the bits decoded at the $m$ th $\mathrm{RN}$ are mapped to a $T$-length symbol vector $\boldsymbol{S}_{\mathrm{r}}^{(m)}(i) \in \mathcal{C}^{1 \times T}$, which is given by

$$
\boldsymbol{S}_{\mathrm{r}}^{(m)}(i)=s(i) \boldsymbol{a}^{(m)}(i) \quad\left(i=1, \cdots, L_{\mathrm{f}}\right),
$$

where according to the input bits $q, \boldsymbol{a}^{(m)}(i)$ is selected from the $Q$ pre-assigned dispersion vectors $\boldsymbol{a}_{q^{\prime}}^{(m)} \in$ $\mathcal{C}^{1 \times T}\left(q^{\prime}=1,2, \cdots, Q\right)$, while $s(i)$ is selected from an $\mathcal{L}$-point PSK/QAM constellation according to the input bits $l$. Similarly to the broadcast phase, $L_{\mathrm{f}}$ denotes the number of space-time blocks successively transmitted in each single transmission frame during the cooperative phase. We note that the input bit-dependent selection of a dispersion vector from a set of $Q$ provides an additional implicit means of transmitting $\log _{2} Q$ bits of information, similarly to the antenna selection philosophy of SM [10]-[12]. To elaborate a little further, the $m$ th relay's bit-to-symbol mapping regime of our cooperative CSTSK scheme employing $Q=4$ and $\mathcal{L}=2$ is shown in Table I. Given $B=\log _{2}(Q \cdot \mathcal{L})$ input bits per block, there are several potential combinations of $Q$ and $\mathcal{L}$, for instance, $(Q, \mathcal{L})=(8,1),(4,2),(2,4)$ and $(1,8)$ for the above-mentioned
TABLE I

EXAMPLE OF $m$ TH RELAY's CSTSK MOdUlation SCHEME, MaPPING 3 Bits Per SPACE-Time Block, With the Aid OF BPSK CONSTELLATION

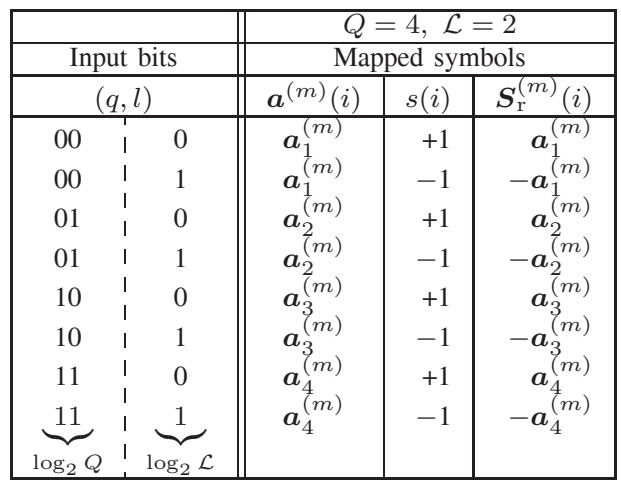

case of conveying $\log _{2}(Q \cdot \mathcal{L})=3$ input bits. ${ }^{4}$

Having generated the STSK-modulated signal vector $S_{\mathrm{r}}^{(m)}(i)$, the corresponding signals received at the DN may be expressed as

$$
\begin{aligned}
\boldsymbol{Y}_{\mathrm{rd}}(i) & =\sum_{m=1}^{M} \alpha_{m} h_{\mathrm{rd}}^{(m)}(i) \boldsymbol{S}_{\mathrm{r}}^{(m)}(i)+\boldsymbol{N}_{\mathrm{d}}^{\prime}(i) \\
& =s(i) \boldsymbol{H}_{\mathrm{rd}}(i) \boldsymbol{A}(i)+\boldsymbol{N}_{\mathrm{d}}^{\prime}(i),
\end{aligned}
$$

where we have

$$
\begin{aligned}
\boldsymbol{H}_{\mathrm{rd}}(i) & =\left[\alpha_{1} h_{\mathrm{rd}}^{(1)}(i), \cdots, \alpha_{M} h_{\mathrm{rd}}^{(M)}(i)\right] \in \mathcal{C}^{1 \times M}, \\
\boldsymbol{A}(i) & =\left[\begin{array}{c}
\boldsymbol{a}^{(1)}(i) \\
\vdots \\
\boldsymbol{a}^{(M)}(i)
\end{array}\right] \in \mathcal{C}^{M \times T}
\end{aligned}
$$

\footnotetext{
${ }^{4}$ Unfortunately, the relaying protocol applicable to our cooperative CSTSK arrangement is limited to the DF scheme. This is because CRC-assisted error detection is implemented at each RN. Furthermore, because the information bits conveyed from the SN during the broadcast phase have to be re-encoded to CSTSK codewords during the cooperative phase, where the PSK/QAM symbol encoded at the SN and the cooperative CSTSK codeword of the RNs do not have a simple amplified relationship.
} 
Here, $\alpha_{m} \in\{1,0\}$ represents the activation/deactivation of the $m$ th relay, where $\alpha_{m}$ is 0 , if any decoding error is identified by the CRC scheme of the $m$ th relay. Otherwise $\alpha_{m}$ is set to 1 . Furthermore, the RN-DN channel coefficients $h_{\mathrm{rd}}^{(m)}(i)(m=$ $1, \cdots, M)$ and the destination's noise components $\boldsymbol{N}_{\mathrm{d}}^{\prime}(i)$ follow the complex-valued Gaussian distributions of $\mathcal{C N}\left(0, \sigma_{\text {rd }}^{2}\right)$ and $\mathcal{C N}\left(0, N_{0}\right)$, respectively. In order to maintain a unity transmission power per time slot, the $M \cdot Q$ pre-assigned dispersion vectors $\boldsymbol{a}_{q}^{(m)}(1 \leq m \leq M, 1 \leq q \leq Q)$ have to satisfy

$$
\operatorname{tr}\left(\boldsymbol{A}_{q^{\prime}} \boldsymbol{A}_{q^{\prime}}^{H}\right)=T\left(q^{\prime}=1, \cdots, Q\right),
$$

where $\operatorname{tr}()$ represents the trace of a matrix and

$$
\boldsymbol{A}_{q^{\prime}}=\left[\begin{array}{c}
\boldsymbol{a}_{q^{\prime}}^{(1)} \\
\vdots \\
\boldsymbol{a}_{q^{\prime}}^{(M)}
\end{array}\right] \in \mathcal{C}^{M \times T} .
$$

We note that the dispersion-matrix set $\boldsymbol{A}_{q^{\prime}}\left(q^{\prime}=1, \cdots, Q\right)$ characterizes our cooperative CSTSK transmission. The design criterion of these matrices will be described in Section IV.

Additionally, the normalized throughput per each of the $b$ time slots or per D-STSK symbol duration for the broadcast phase is $R_{1}$ and that of the cooperative phase is $R_{2}$, which are given by

$$
\begin{array}{ll}
R_{1}=\log _{2} \mathcal{L}^{\prime} & {[\mathrm{bits} / \mathrm{symbol}]} \\
R_{2}=\frac{\log _{2}(Q \cdot \mathcal{L})}{T} & {[\mathrm{bits} / \mathrm{symbol}],}
\end{array}
$$

leading to the total normalized throughput of

$$
R=\frac{b R_{1}+T R_{2}}{2(b+T)}=\frac{\log _{2}(Q \cdot \mathcal{L})}{b+T}[\mathrm{bits} / \mathrm{symbol}]
$$

It can be seen from (11) that upon increasing either the number of dispersion vectors $Q$ or the classic PSK/QAM constellation size $\mathcal{L}$, the transmission rate of the cooperative phase increases.

Moreover, the maximum achievable transmit diversity order of the cooperative CSTSK scheme is upper-bounded by $\min (M, T)$, according to the well-known pairwise-error probability analysis based on the Chernoff upper bound [17]. As mentioned above, the proposed cooperative CSTSK scheme is capable of supporting an arbitrary number of RNs and of adopting diverse modulation schemes, while striking a flexible tradeoff between diversity and throughput. Therefore, this architecture becomes especially beneficial in a scenario, where the network topology and/or the channel conditions fluctuate rapidly.

\section{Cooperative Asynchronous CSTSK Arrangement}

It is implied in (5) that the RNs typically have to synchronize with each other within a fraction of the symbol duration, owing to the requirement of their simultaneous transmissions. However, as mentioned above, it is a challenging task to acquire accurate IRS. Therefore, in order to relax this IRSrelated limitation, hereby we impose a further constraint on the $Q$ dispersion matrices of $\boldsymbol{A}_{q^{\prime}}\left(q^{\prime}=1, \cdots, Q\right)$ in addition to the power constraint of (8). More specifically, $\boldsymbol{A}_{q^{\prime}}$ is generated by ensuring that in each column of $\boldsymbol{A}_{q^{\prime}}$ only one component has a complex non-zero value, while the others become zero. By obeying this constraint, only one of the $M$ RNs transmits its signal during each symbol interval, and hence we can avoid the requirement of symbol-synchronized simultaneous relay transmissions. ${ }^{5}$

To elaborate a little further, as explicitly mentioned in [12], the SM scheme developed for co-located MIMO arrangements enables the transmitter to dispense with symbol-level IAS, since in the SM scheme only a single AE is activated during each symbol duration. Similarly, due to the abovementioned constraint of imposing a sparse structure on the dispersion matrices of our cooperative Asynchronous CSTSK (ACSTSK) scheme, only a single RN is activated within each symbol duration, hence exhibiting robustness against IRS errors provided that they are limited to a fraction of the symbol-duration. We note that the potential IRS error may severely degrade the performance of conventional cooperative STCs, which require symbol-level IRS, as investigated in [4]. ${ }^{6}$ On the other hand, when the IRS error exceeds the symbol duration, a severe impairment may be imposed also on our cooperative ACSTSK scheme, since the structure of the STSK codeword is destroyed. In order to mitigate the performance erosion associated with high IRS errors, we may be able to incorporate the Loosely-Synchronized (LS)-code aided SpaceTime Spreading (STS) technique of [7] into our cooperative STSK scheme, although the detailed investigations will be left for our future studies owing to space-limitations. As a further means of reducing the effects imposed by the high IRS, it may be beneficial to employ multi-carrier transmissions, which extends the symbol durations commensurately with the number of carriers.

\section{IEI-Free Joint ML Detection at the Destination Receiver}

At the DN, the directly transmitted signals of (2) and the relayed signals of (5) are jointly detected using a lowcomplexity single-stream ML detector. ${ }^{7} 8$

Firstly, by applying the vectorial stacking operation $\operatorname{vec}()$ to both sides of (5), we arrive at the linearized relay-destination system's output in the form of [17]

$$
\overline{\boldsymbol{Y}}_{\mathrm{rd}}(i)=\overline{\boldsymbol{H}}_{\mathrm{rd}}(i) \chi \boldsymbol{K}(i)+\overline{\boldsymbol{N}}_{\mathrm{d}}^{\prime}(i),
$$

\footnotetext{
${ }^{5}$ Interestingly, the well-known repetition-based cooperation [1], [18] may be viewed as a special case of our ACSTSK scheme, where we have $M=T$, $Q=1$ and $\boldsymbol{A}_{1}=\boldsymbol{I}_{M}$. Here, in the repetition-based cooperation the single dispersion matrix $\boldsymbol{A}_{1}$ is the identity matrix $\boldsymbol{I}$, while the cooperative ACSTSK scheme can have arbitrary non-zero complex values in each dispersion matrix under the norm constraint, hence having a higher degree of design freedom in comparison to its repetition-based cooperation aided counterpart.

${ }^{6}$ Since the cooperative CSTSK arrangement requires the RNs' simultaneous transmissions similarly to the conventional cooperative STCs, the IRS error naturally degrades its achievable performance, according to [4].

${ }^{7}$ In [13] the IEI-free optimal ML detector was presented for point-to-point co-located MIMO scenarios. Here, we extend it to the cooperative STSK receiver, where the direct SD link and the cooperative STSK relaying links are jointly detected, in order to attain a good BER performance without imposing a prohibitively high complexity.

${ }^{8}$ Recently, a further decoding algorithm was developed for co-located STSK scenarios in [19], which may be readily applied in our cooperative STSK arrangement. However, the detailed investigation of this idea is beyond the scope of this paper.
} 
where we have

$$
\begin{array}{rlrl}
\overline{\boldsymbol{Y}}_{\mathrm{rd}}(i) & =\operatorname{vec}\left[\boldsymbol{Y}_{\mathrm{rd}}(i)\right] & & \in \mathcal{C}^{T \times 1}, \\
\overline{\boldsymbol{H}}_{\mathrm{rd}}(i) & =\boldsymbol{I} \otimes \boldsymbol{H}_{\mathrm{rd}}(i) & & \in \mathcal{C}^{T \times M T}, \\
\overline{\boldsymbol{N}}_{\mathrm{d}}^{\prime}(i) & =\operatorname{vec}\left[\boldsymbol{N}_{\mathrm{d}}^{\prime}(i)\right] & \in \mathcal{C}^{T \times 1}, \\
\boldsymbol{\chi} & =\left[\operatorname{vec}\left(\boldsymbol{A}_{1}\right), \cdots, \operatorname{vec}\left(\boldsymbol{A}_{Q}\right)\right] & \in \mathcal{C}^{M T \times Q},
\end{array}
$$

and

$$
\boldsymbol{K}(i)=[\underbrace{0, \cdots, 0}_{q-1}, s(i), \underbrace{0, \cdots, 0}_{Q-q}]^{T} \in \mathcal{C}^{Q \times 1} .
$$

Furthermore, $\boldsymbol{I}$ is the identity matrix and $\otimes$ is the Kronecker product. It is worth mentioning that the linearized relaydestination system model of (13) does not contain any IEI, because the equivalent signal vector $\boldsymbol{K}(i)$ has only a single non-zero symbol component, similarly to SM [10]-[12].

Finally, the joint system model, combining the broadcast phase of (2) and the cooperative phase of (13), may be formulated as

$$
\begin{aligned}
\hat{\boldsymbol{Y}}(i) & =\left[\begin{array}{c}
\boldsymbol{Y}_{\mathrm{sd}}(i) \\
\overline{\boldsymbol{Y}}_{\mathrm{rd}}(i)
\end{array}\right] \in \mathcal{C}^{(b+T) \times 1} \\
& =\hat{\boldsymbol{H}}(i) \hat{\boldsymbol{S}}(i)+\hat{\boldsymbol{N}}(i),
\end{aligned}
$$

where we have

$$
\begin{aligned}
\hat{\boldsymbol{H}}(i) & =\left[\begin{array}{c:c}
h_{\mathrm{sd}}(i) \boldsymbol{I}_{b} & \mathbf{0} \\
\hdashline \mathbf{0} & \boldsymbol{H}_{\mathrm{rd}}(i) \bar{\chi}
\end{array}\right] \in \mathcal{C}^{(b+T) \times(b+Q)}, \\
\hat{\boldsymbol{S}}(i) & =\left[\begin{array}{c}
\boldsymbol{S}_{\mathrm{s}}(i) \\
\boldsymbol{K}(i)
\end{array}\right] \in \mathcal{C}^{(b+Q) \times 1}, \\
\hat{\boldsymbol{N}}(i) & =\left[\begin{array}{c}
\boldsymbol{N}_{\mathrm{d}}(i) \\
\boldsymbol{N}^{\prime}(i)
\end{array}\right] \in \mathcal{C}^{(b+T) \times 1} .
\end{aligned}
$$

Let us then consider the conditional probability of

$$
\begin{aligned}
& P\left(\hat{\boldsymbol{Y}}(i) \mid \hat{\boldsymbol{H}}(i), \hat{\boldsymbol{S}}^{(q, l)}\right) \\
& =\frac{1}{\left(\pi N_{0}\right)^{b+T}} \exp \left(-\frac{\left\|\hat{\boldsymbol{Y}}(i)-\hat{\boldsymbol{H}}(i) \hat{\boldsymbol{S}}^{(q, l)}\right\|^{2}}{N_{0}}\right),
\end{aligned}
$$

where

$$
\hat{\boldsymbol{S}}^{(q, l)}=\left[\begin{array}{c}
\boldsymbol{S}_{\mathbf{s}}^{(q, l)} \\
\boldsymbol{K}^{(q, l)}
\end{array}\right] \in \mathcal{C}^{(b+Q) \times 1}
$$

with

$$
\boldsymbol{K}^{(q, l)}=[\underbrace{0, \cdots, 0}_{q-1}, s_{l}, \underbrace{0, \cdots, 0}_{Q-q}]^{T} .
$$

Here, $s_{l}$ denotes the $l$ th constellation point of $\mathcal{L}$-PSK/QAM, employed during the cooperative phase and $\boldsymbol{S}_{\mathrm{s}}^{(q, l)}$ represents the modulated symbols of the broadcast phase, corresponding to the bits of the set $(q, l)$.

Then, the optimal ML detector of our cooperative CSTSK scheme may be formulated with the assistance of [11] as

$$
\begin{aligned}
(\hat{q}, \hat{l}) & =\arg \max _{q, l} P\left(\hat{\boldsymbol{Y}}(i) \mid \hat{\boldsymbol{H}}(i), \hat{\boldsymbol{S}}^{(q, l)}\right) \\
& =\arg \min _{q, l}\left\|\hat{\boldsymbol{Y}}(i)-\hat{\boldsymbol{H}}(i) \hat{\boldsymbol{S}}^{(q, l)}\right\|^{2} \\
& =\arg \min _{q, l}\left(\left\|\boldsymbol{Y}_{\mathrm{sd}}(i)-h_{\mathrm{sd}}(i) \boldsymbol{S}_{\mathrm{s}}^{(q, l)}\right\|^{2}\right. \\
& \left.+\left\|\overline{\boldsymbol{Y}}_{\mathrm{rd}}(i)-s_{l}\left(\overline{\boldsymbol{H}}_{\mathrm{rd}}(i) \boldsymbol{\chi}\right)_{q}\right\|^{2}\right),
\end{aligned}
$$

where $\left(\overline{\boldsymbol{H}}_{\mathrm{rd}}(i) \boldsymbol{\chi}\right)_{q}$ is the $q$ th column of $\overline{\boldsymbol{H}}_{\mathrm{rd}}(i) \boldsymbol{\chi}$. The first term of (29) indicates the detection of the source-destination signals, while the second term corresponds to that of the relay-destination signals, where all the signal components are independent of each other and hence no IEI is imposed.

The computational complexity per bit imposed by calculating (29) may be evaluated in terms of the number of realvalued multiplications, which is given by

$$
\frac{4 M Q T^{2}+6 T Q \mathcal{L}+4 \mathcal{L}^{\prime}+2 b Q \mathcal{L}}{\log _{2}(Q \cdot \mathcal{L})} .
$$

Furthermore, that of the cooperative ACSTSK scheme may be simplified to

$$
\frac{4 Q T^{2}+6 T Q \mathcal{L}+4 \mathcal{L}^{\prime}+2 b Q \mathcal{L}}{\log _{2}(Q \cdot \mathcal{L})}
$$

This complexity is as low as those of the OSTBC [16] and SM schemes [11] used in an identical cooperative scenario. In the rest of this paper, we employ the parameter-based system notation of the cooperative $\operatorname{CSTSK}(M, T, Q)$ for the cooperative phase.

\section{System Model of Cooperative DSTSK Scheme}

Having introduced our cooperative CSTSK scheme in Section II, we now conceive its differentially-encoded counterpart. Here, we aim for designing a simplified cooperative system, dispensing with any CSI estimation at the nodes, while retaining the fundamental benefits of the above-mentioned cooperative CSTSK scheme.

Fig. 2 shows the schematic of our cooperative DSTSK system, which was developed from the co-located DSTSK scheme of [13] and from the cooperative CSTSK scheme of Fig. 1. More specifically, in our cooperative DSTSK scheme, the classic $\mathcal{L}^{\prime}$-point Differential PSK (DPSK) modulation scheme is employed at the SN, instead of the PSK/QAM scheme of the cooperative CSTSK arrangement seen in Fig. 1. To be specific, $\mathcal{L}^{\prime}$-PSK symbols $\boldsymbol{S}_{\mathrm{S}}(i)=\left[s_{1}(i), \cdots, s_{b}(i)\right]^{T}$ are differentially encoded in order to yield the symbols of $\boldsymbol{S}_{\mathrm{S}}^{\prime}(i)=\left[s_{1}^{\prime}(i), \cdots, s_{b}^{\prime}(i)\right]^{T}$ according to $s_{j}^{\prime}(i)=s_{j}(i) s_{j}^{\prime}(i-$ 1) $\left(j=1, \cdots, b, i=1, \cdots, L_{\mathrm{f}}\right)$. This enables the RNs and the DN to decode their symbols without having access to the corresponding CSI.

Moreover, as shown in Fig. 2, we employed the DSTSKencoding principle at each RN, instead of the CSTSKencoding principle of the cooperative CSTSK scheme seen in Fig. 1. To be more specific, at the $m$ th RN, the $b \log _{2} \mathcal{L}^{\prime}$ bits per $b$-slot block, which are received during the broadcast phase and are decoded correctly, are S/P converted to $B_{1}=\log _{2} \mathcal{L}$ and $B_{2}=\log _{2} Q$ bits, similarly to the cooperative CSTSK scheme of Fig. 1. Then, the $\mathcal{L}$-PAM symbol $s(i)=s_{l}$ is mapped according to $B_{1}=\log _{2} \mathcal{L}$, while one out of the $Q$ Hermitian dispersion matrices $\boldsymbol{A}(i)=\boldsymbol{A}_{q}$ is activated according to $B_{2}=\log _{2} Q$ bits. The space-time signals $\tilde{\boldsymbol{X}}(i)=$ $s(i) \boldsymbol{A}(i)$ are then uniquely and unambiguously mapped to the unitary matrix $\boldsymbol{X}(i)$ using the Cayley transform of [13]

$$
\boldsymbol{X}(i)=[\boldsymbol{I}-j \tilde{\boldsymbol{X}}(i)][\boldsymbol{I}+j \tilde{\boldsymbol{X}}(i)]^{-1} \quad\left(i=1, \cdots, L_{\mathrm{f}}\right) .
$$




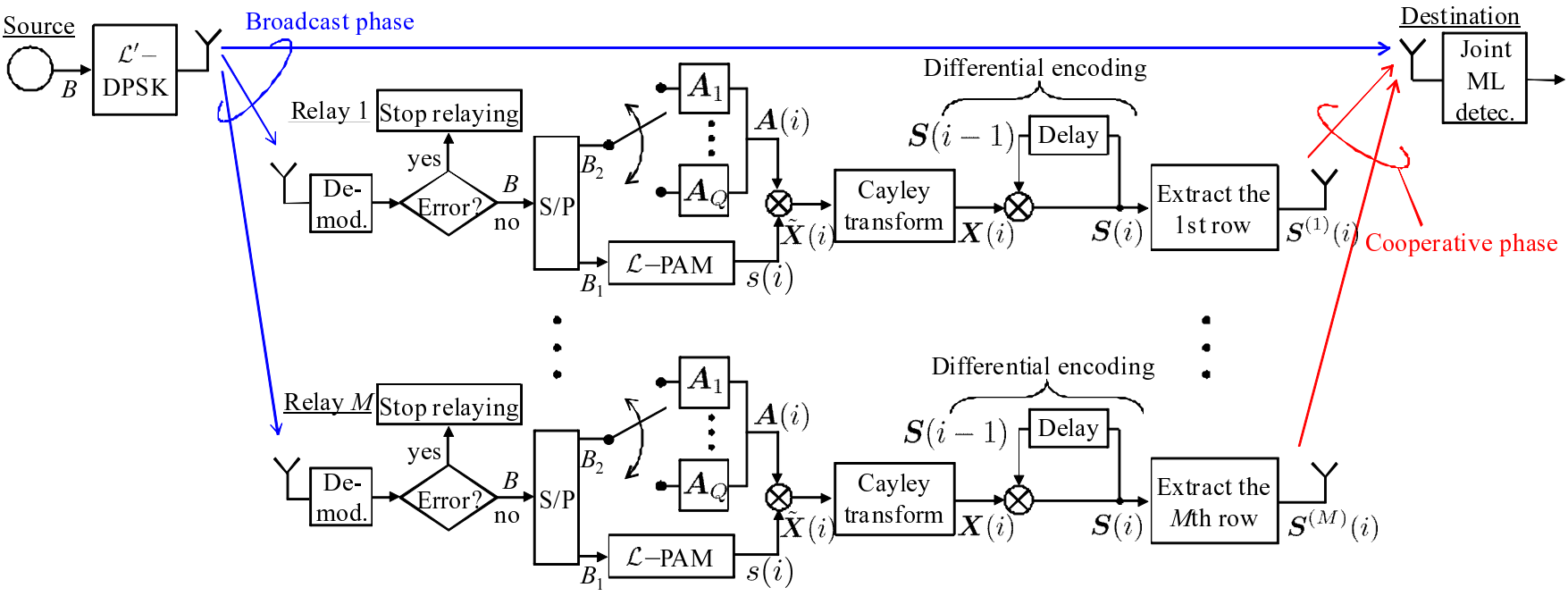

Fig. 2. Schematic of our cooperative DSTSK scheme assisted by selective DF relaying.

Next, the differential encoding operation is carried out as follows:

$$
\boldsymbol{S}_{\mathrm{r}}^{(m)}(i)=\boldsymbol{S}_{\mathrm{r}}^{(m)}(i-1) \boldsymbol{X}(i) \quad\left(i=1, \cdots, L_{\mathrm{f}}\right),
$$

assuming that the initial codeword was $\boldsymbol{S}_{\mathrm{r}}^{(m)}(0)=\boldsymbol{I}$. Finally, the $m$ th-row components of the signals $\boldsymbol{S}_{\mathrm{r}}^{(m)}(i)$ are transmitted from the $m$ th RN over $T$ symbol durations. Here, we note that in order to enable the differential encoding operation of (33), the relationship of $M=T$ has to be satisfied in our cooperative DSTSK scheme.

Similarly to (4), the signals received at the DN during the cooperative phase may be expressed as

$$
\begin{aligned}
\boldsymbol{Y}_{\mathrm{rd}}(i) & =\sum_{m=1}^{M} \alpha_{m} h_{\mathrm{rd}}^{(m)}(i) \boldsymbol{S}_{\mathrm{r}}^{(m)}(i)+\boldsymbol{N}_{\mathrm{d}}^{\prime}(i) \\
& =\boldsymbol{H}_{\mathrm{rd}}(i) \boldsymbol{S}_{\mathrm{r}}^{(m)}(i)+\boldsymbol{N}_{\mathrm{d}}^{\prime}(i),
\end{aligned}
$$

where $\alpha_{m}$ indicates the activation or deactivation of the $m$ th $\mathrm{RN}$, while $\boldsymbol{N}_{\mathrm{d}}^{\prime}(i)$ denotes the complex valued Gaussian variables obeying the distribution of $\mathcal{C N}\left(0, N_{0}\right)$. Furthermore, the equivalent channel matrix $\boldsymbol{H}_{\mathrm{rd}}(i)$ is given by (6).

\section{A. Cooperative Asynchronous DSTSK Scheme}

Similarly to the co-located ACSTSK scheme of Section II-C employing a specific dispersion-matrix structure, in this section we conceive a cooperative Asynchronous DSTSK (ADSTSK) scheme. More specifically, a real-valued diagonal constraint may be imposed on the $Q$ dispersion matrices of $\boldsymbol{A}_{q^{\prime}}\left(q^{\prime}=1, \cdots, Q\right)$. Due to this constraint, the DSTSK codewords $\boldsymbol{S}_{\mathrm{r}}^{(m)}(i)$ of (33) as well as the unitary matrix $\boldsymbol{X}(i)$ of (32) maintain a diagonal structure. Hence only one of the $M$ RNs transmits its signal during each symbol interval. As the benefit of this diagonal constraint, we can avoid the requirement of perfectly-synchronized simultaneous relay transmissions.

\section{B. IEI-Free Joint ML Detection at the Destination Receiver}

Similarly to the IEI-free joint ML detection derived for the cooperative CSTSK scheme in Section II-D, we introduce that of the cooperative DSTSK scheme in this section.
Firstly, the equivalent signals received at the DN during the broadcast phase of Fig. 2 may be formulated with the aid of the differential-decoding operation, as

$$
\begin{aligned}
\boldsymbol{Y}_{\mathrm{sd}}^{\prime}(i) & =\operatorname{diag}\left[\boldsymbol{Y}_{\mathrm{sd}}^{*}(i-1) \cdot \boldsymbol{Y}_{\mathrm{sd}}^{\mathrm{T}}(i)\right] \\
& =h_{\mathrm{sd}} \boldsymbol{S}_{\mathrm{S}}(i)+\hat{\boldsymbol{N}}_{\mathrm{d}}(i)
\end{aligned}
$$

where we assumed that the source-destination channel remains constant over $b$ symbol durations, while each component of $\hat{\mathbf{N}}_{\mathrm{d}}(i)$ obeys the Gaussian distribution of $\mathcal{C N}\left(0,2 N_{0}\right)$. By contrast, assuming that the fading channel's envelope remains constant over the two DSTSK block durations $2 T$, the corresponding signal block of (35) received at the DN during the cooperative phase $\boldsymbol{Y}_{\mathrm{rd}}(i)$ is modified to

$$
\begin{aligned}
\boldsymbol{Y}_{\mathrm{rd}}(i) & =\boldsymbol{H}_{\mathrm{rd}} \boldsymbol{S}_{\mathrm{r}}^{(m)}(i)+\boldsymbol{N}_{\mathrm{d}}^{\prime}(i) \\
& =\boldsymbol{Y}_{\mathrm{rd}}(i-1) \boldsymbol{X}(i)+\boldsymbol{N}_{\mathrm{d}}^{\prime}(i)-\boldsymbol{N}_{\mathrm{d}}^{\prime}(i-1) \boldsymbol{X}(i),
\end{aligned}
$$

which does not include any channel components. Instead of directly applying optimum ML detection to the received signal of (38), we introduce the linearization technique of [20] for the sake of facilitating the employment of the single-stream-based ML detector of (29). More specifically, upon multiplying both sides of (38) by $[\boldsymbol{I}+j \tilde{\boldsymbol{X}}(i)]$, we arrive at

$$
\begin{gathered}
\underbrace{\boldsymbol{Y}_{\mathrm{rd}}(i)-\boldsymbol{Y}_{\mathrm{rd}}(i-1)}_{\hat{\boldsymbol{Y}}_{\mathrm{rd}}(i)}=\underbrace{-j\left[\boldsymbol{Y}_{\mathrm{rd}}(i)+\boldsymbol{Y}_{\mathrm{rd}}(i-1)\right]}_{\hat{\boldsymbol{H}}_{\mathrm{rd}}(i)} \tilde{\boldsymbol{X}}(i) \\
+\underbrace{\{-\boldsymbol{V}(i)[\boldsymbol{I}+j \tilde{\boldsymbol{X}}(i)]-\boldsymbol{V}(i-1)[\boldsymbol{I}-j \tilde{\boldsymbol{X}}(i)]\}}_{\hat{\boldsymbol{N}}_{\mathrm{d}}^{\prime}(i)}
\end{gathered}
$$

where $\hat{\boldsymbol{Y}}_{\text {rd }}(i)$ and $\hat{\boldsymbol{H}}_{\mathrm{rd}}(i)$ represent the equivalent received signals and the equivalent channel matrix, while the equivalent noise matrix $\hat{N}_{\mathrm{d}}^{\prime}(i)$ has independent columns with a covariance of

$$
\hat{N}_{0}=N_{0}\left(\boldsymbol{I}+\tilde{\boldsymbol{X}}^{2}(i)\right) .
$$


TABLE II

Basic System Parameters of the Cooperative CSTSK $(M, T, Q)$ and DSTSK $(M, T, Q) \operatorname{SchemeS}$

\begin{tabular}{|c|c|}
\hline \multicolumn{2}{|c|}{ CSTSK } \\
\hline Number of relay nodes & $M=2-4$ \\
\hline Number of receive antennas at each node & $N=1$ \\
\hline Symbol durations per block & $T=2,3$ \\
\hline Number of dispersion matrices & $Q=2,4,8,16$ \\
\hline Modulation during broadcast phase & $\mathcal{L}^{\prime}-\mathrm{PSK} / \mathrm{QAM}$ \\
\hline Modulation during cooperative phase & $\mathcal{L}-\mathrm{PSK} / \mathrm{QAM}$ \\
\hline Relaying scheme & CRC-activated selective DF scheme \\
\hline Channels & Frequency-flat Rayleigh fading \\
\hline Channel's coherence-time & $\tau=1$ block duration \\
\hline Geometrical channel gains & $\left(\sigma_{\mathrm{sd}}^{2}, \sigma_{\mathrm{sr}}^{2}, \sigma_{\mathrm{rd}}^{2}\right)=(1,4,2)$ \\
\hline Detector & Joint ML detector of (29) \\
\hline \multicolumn{2}{|c|}{ DSTSK } \\
\hline Number of relay nodes & $M=2$ \\
\hline Number of receive antennas at each node & $N=1$ \\
\hline Symbol durations per block & $T=M$ \\
\hline Number of dispersion matrices & $Q=2,4,8$ \\
\hline Modulation during broadcast phase & $\mathcal{L}^{\prime}-$ DPSK \\
\hline Modulation during cooperative phase & $\mathcal{L}-$ PAM \\
\hline Relaying scheme & CRC-activated selective DF scheme \\
\hline Channels & Frequency-flat Rayleigh fading \\
\hline Channel's coherence-time & $\tau=2$ block durations \\
\hline Geometrical channel gains & $\left(\sigma_{\mathrm{sd}}^{2}, \sigma_{\mathrm{sr}}^{2}, \sigma_{\mathrm{rd}}^{2}\right)=(1,4,2)$ \\
\hline Detector & Joint ML detector of (29) \\
\hline
\end{tabular}

Finally, by applying the $\operatorname{vec}($ ) operation to (39), we arrive at [20]

$$
\tilde{\boldsymbol{Y}}_{\mathrm{rd}}(i)=\tilde{\boldsymbol{H}}_{\mathrm{rd}}(i) \chi \boldsymbol{K}(i)+\tilde{\boldsymbol{N}}_{\mathrm{d}}^{\prime}(i)
$$

where we have

$$
\begin{aligned}
\tilde{\boldsymbol{Y}}_{\mathrm{rd}}(i) & =\operatorname{vec}\left[\hat{\boldsymbol{Y}}_{\mathrm{rd}}(i)\right] \in \mathcal{C}^{N T \times 1}, \\
\tilde{\boldsymbol{H}}_{\mathrm{rd}}(i) & =\boldsymbol{I} \otimes \hat{\boldsymbol{H}}_{\mathrm{rd}}(i) \in \mathcal{C}^{N T \times M T} \\
\tilde{\boldsymbol{N}}_{\mathrm{d}}^{\prime}(i) & =\operatorname{vec}\left[\hat{\boldsymbol{N}}_{\mathrm{d}}^{\prime}(i)\right] \in \mathcal{C}^{N T \times 1},
\end{aligned}
$$

while $\chi$ and $\boldsymbol{K}(i)$ are given by (17) and (18), respectively, in the same manner as the cooperative CSTSK scheme of Section II.

The cooperative DSTSK scheme's equivalent signals of (37) and (41) received at the DN exhibit the same structures as for those of the cooperative CSTSK scheme formulated in (2) and (13). Therefore, the single-stream-based joint ML detection algorithm of (29) developed for the cooperative CSTSK scheme may also readily be invoked for our cooperative DSTSK scheme, acknowledging that the resultant cooperative DSTSK scheme's performance would inevitably suffer from the usual SNR loss imposed by differential encoding.

In the rest of this paper, our DSTSK scheme is characterized as ' $\operatorname{DSTSK}(M, T, Q)$ ', similarly to the $\operatorname{CSTSK}(M, T, Q)$ scheme.

\section{Performance Results}

In this section we provide our performance results, comparing different DF scenarios as well as different cooperative schemes. In line with [7], we considered independent Rayleigh block-fading environments, having the geometrical distancereduction based channel gains of $\sigma_{\mathrm{sd}}^{2}=1, \sigma_{\mathrm{sr}}^{2}=4$ and $\sigma_{\text {rd }}^{2}=2$, which remains constant over $(b+T)$ STSK symbol durations. Furthermore, the SN was assumed to employ QPSK modulation, which indicates $\mathcal{L}^{\prime}=4 .^{9}$

According to the previous studies of Linear Dispersion Codes (LDCs) [21], there exists several potential approaches to the optimization of the dispersion matrix set $\boldsymbol{A}_{q^{\prime}}\left(q^{\prime}=\right.$ $1, \cdots, Q)$, such as the capacity maximization criterion [22] and pairwise error probability minimization [23]. In this contribution, we employ the well-known rank- and determinantcriterion of [24] in order to attain the maximum achievable diversity order as well as a high coding gain. The dispersionvector sets, which were obtained by random search and were employed in our simulations, are shown in the Appendix. The basic system parameters employed for our simulations are listed in Table II.

Fig. 3 shows the achievable BER performance of our cooperative CSTSK $(2,2,4)$ scheme, employing QPSK modulation both at the $\mathrm{SN}$ and at the RNs, where the normalized transmission rate was $R=1.0 \mathrm{bits} / \mathrm{symbol}$. Here, we compared three different DF schemes, namely the perfect decision based $\mathrm{DF}$, the conventional DF and the proposed CRC-activated DF schemes, where the perfect DF scheme assumed having perfect source-relay channels, hence imposing no errors by the relays' decoders, while in the conventional DF scheme all the $M$ RNs were assumed to join the cooperative transmission regime, regardless of the presence or absence of decoding errors. We also plotted the BER curve of the corresponding non-cooperative scenario, assuming the employment of BPSK modulation at the SN. Observe in Fig. 3 that the perfect and the selective DF schemes attained a transmit diversity

\footnotetext{
${ }^{9}$ To elaborate a little further, we also simulated further geometrical-distance scenarios, such as $\sigma_{\mathrm{sd}}^{2}=1, \sigma_{\mathrm{sr}}^{2}=1$ and $\sigma_{\mathrm{rd}}^{2}=1$. As the results, it was found that although the corresponding BER curves in Figs. 3-9 were shifted to a higher SNR direction due to the reduced channel gains, the relative performance between each cooperative scheme as well as the maximum achievable diversity order was not affected. We note that these are not explicitly shown in this paper for reasons of space economy.
} 

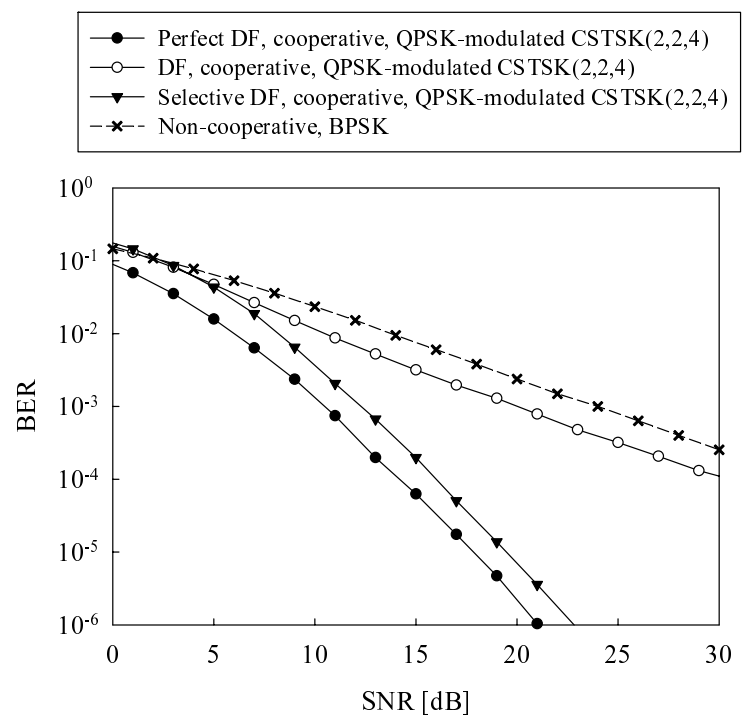

Fig. 3. Achievable BER performance of our QPSK-modulated cooperative $\operatorname{CSTSK}(2,2,4)$ system, comparing different DF relaying schemes, such as the perfect DF scheme having no information loss, the conventional DF scheme and the CRC-activated DF scheme. We also characterized the non-cooperative scenario employing BPSK modulation.
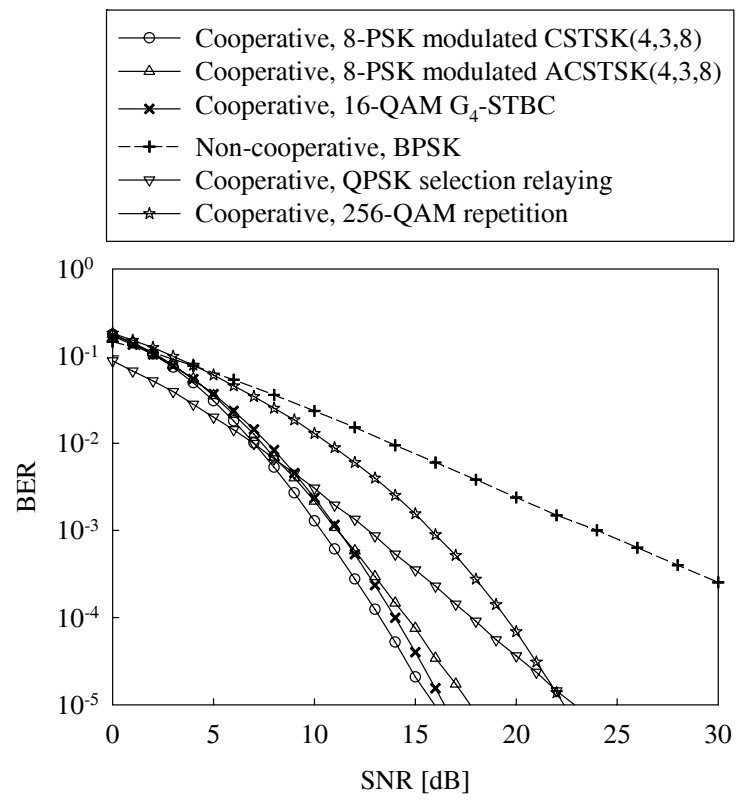

Fig. 4. Achievable BER performance of our $\mathcal{L}=8$ PSK-modulated cooperative $\operatorname{CSTSK}(4,3,8)$ and $\mathcal{L}=8$ PSK-modulated cooperative $\operatorname{ACSTSK}(4,3,8)$ schemes obeying the architecture of Fig. 1, while $\mathcal{L}^{\prime}=4$ PSK was employed for the SN. The corresponding BER curves of the cooperative $\mathrm{G}_{4}$ STBC scheme as well as of the BPSK-modulated non-cooperative scheme were also calculated as benchmarkers. For the sake of further performance comparison, the QPSK-modulated selection relaying scheme [18] and the 256QAM assisted repetition-based cooperation scheme [1] were also considered. Here, each scheme exhibited a normalized transmission rate of $R=1.0$ bits/symbol.

order of three, hence both outperformed the conventional DF scheme and the non-cooperative scenario. Here, it should be emphasized that the selective DF scheme achieved a reducedoverhead distributed operation at each RN. On the other hand, the BER curves of the conventional DF scheme and of the noncooperative scenario did not exhibit any additional transmit diversity gain.

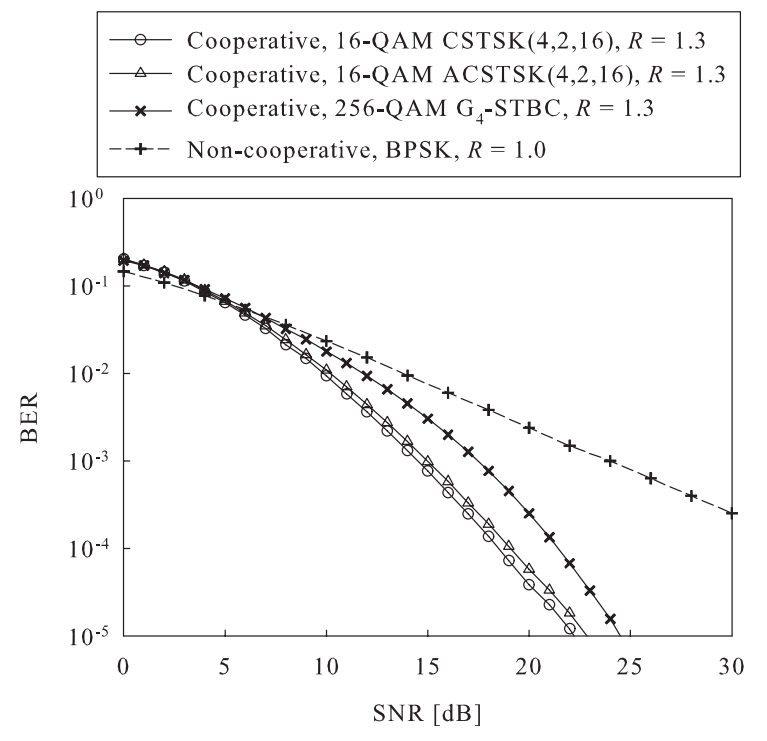

Fig. 5. Achievable BER performance of our $\mathcal{L}=16$ QAM cooperative $\operatorname{CSTSK}(4,2,16)$ and $\mathcal{L}=16$ QAM cooperative $\operatorname{ACSTSK}(4,2,16)$ schemes obeying the architecture of Fig. 1 , while $\mathcal{L}^{\prime}=4$ PSK was employed for the $\mathrm{SN}$. The corresponding BER curves of the $\mathcal{L}=256$ QAM cooperative $\mathrm{G}_{4}$ STBC scheme as well as BPSK-modulated non-cooperative scheme were also calculated as benchmarkers. Here, each of the cooperative schemes exhibited a normalized transmission rate of $R=1.3 \mathrm{bits} / \mathrm{symbol}$.
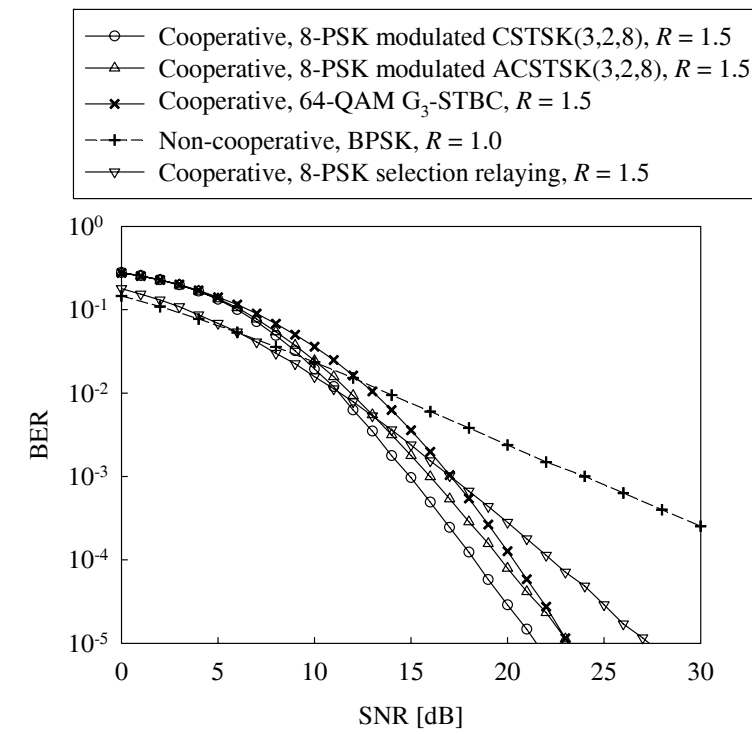

Fig. 6. Achievable BER performance of our $\mathcal{L}=8$ PSK-modulated cooperative $\operatorname{CSTSK}(3,2,8)$ and $\operatorname{ACSTSK}(3,2,8)$ schemes obeying the architecture of Fig. 1, while $\mathcal{L}^{\prime}=8$ PSK was employed for the SN. The corresponding BER curves of the $\mathcal{L}=64$ QAM cooperative $\mathrm{G}_{3}$-STBC scheme as well as BPSKmodulated non-cooperative scheme were also calculated as benchmarkers. Furthermore, the 8-PSK modulated selection relaying scheme [18] was also considered. Here, each of the cooperative schemes exhibited a normalized transmission rate of $R=1.5$ bits/symbol.

In order to provide further insights, in Figs. 4, 5 and 6 we compared our cooperative CSTSK and ACSTSK schemes to the cooperative OSTBC arrangements [16], having the corresponding bandwidth efficiency. Here, we CRC-activated selective DF relaying for all the simulated scenarios. Fig. 4 investigated the scenario of a normalized transmission rate of $R=1.0 \mathrm{bits} / \mathrm{symbol}$, where $M=4 \mathrm{RNs}$ were considered. Observe in Fig. 4 that the three space-time cooperation aided 


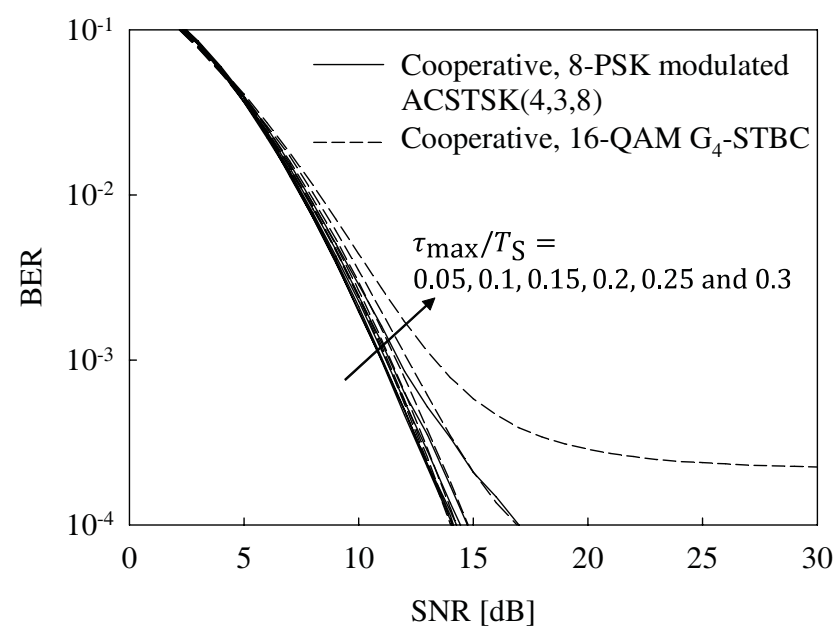

(a) $\beta=0.5$

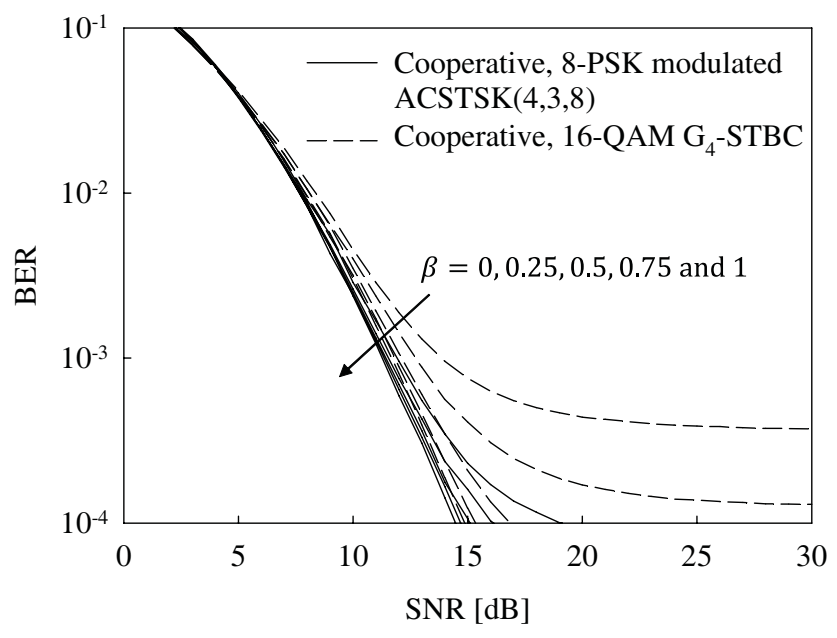

(b) $\tau_{\max }=0.25 T_{\mathrm{S}}$

Fig. 7. Achievable BER performance of the cooperative ACSTSK and the cooperative OSTBC schemes simulated in Fig. 4, where we considered a RN-specific IRS error $\tau_{\max }$ and the raised-cosine Nyquist filter having the roll-off factor $\beta$, which was employed at the SN and the RNs.

schemes, namely the CSTSK, the ACSTSK and the OSTBC schemes, achieved a useful diversity gains in comparison to the non-cooperative scenario. The cooperative CSTSK scheme outperformed the cooperative OSTBC scheme, as predicted from the results characterized by the co-located CSTSK arrangements [13]. Additionally, the cooperative ACSTSK scheme exhibited a slightly lower performance than those of the cooperative CSTSK and OSTBC schemes, due to the restricted dispersion matrix structure discussed in Section II-C. Nevertheless, the cooperative ACSTSK scheme's benefit of dispensing with symbol-level IRS may be especially useful for the scenario suffering from a rapid topology change, where perfect IRS is hard to achieve. ${ }^{10}$

In order to provide further insights, in Fig. 4 we also included the corresponding BER curves of the conventional relay selection aided scheme [18] as well as the repetition-based cooperation scheme [1]. In the selection relaying scheme, a single RN having the highest source-relay channel amplitude is selected out of $M=4 \mathrm{RNs}$, while in the repetitionbased cooperation scheme each of the $M=4$ RNs actively relays the re-encoded symbol during a time slot allocated to the respective RN. It was found in Fig. 4 that the relay selection aided scheme did not attain the maximum achievable diversity order of four, while the repetition-based cooperation scheme was outperformed by the other space-time cooperative schemes due to its inefficiency.

Furthermore, when increasing the normalized transmission rate $R$, the performance advantage of our CSTSK and ACSTSK schemes becomes more explicit, as observed in Figs. 5 and 6 , which correspond to the scenarios of the normalized transmission rates of $R=1.3 \mathrm{bits} / \mathrm{symbol}$ and of $R=1.5$ bits/symbol. To expound a little further, it was found in Figs. 5

\footnotetext{
${ }^{10}$ Additionally, we also investigated the equal geographical-gain scenario of $\left(\sigma_{\mathrm{sd}}^{2}, \sigma_{\mathrm{sr}}^{2}, \sigma_{\mathrm{rd}}^{2}\right)=(1,1,1)$, although the results are not included here for reasons of space-economy. It was found that although the corresponding BER curves were shifted toward higher SNRs owing to the reduced channel gains, the fundamental characteristics, such as the maximum achievable diversity order as well as the BER rank-order of the cooperative STSK schemes and of the other cooperative benchmarkers did not change.
}

and 6 that our CSTSK and ACSTSK schemes outperformed the corresponding BER of the cooperative OSTBC scheme. This is mainly owing to the fact that the cooperative OSTBC scheme is typically required to employ power-hungry high order modulation, in order to attain an increased transmission rate.

In Fig. 7, we investigated the effects of IRS errors on the BER performance of the cooperative ACSTSK and the cooperative OSTBC, which was previously shown in Fig. 4. Here, we introduced a RN-specific random IRS error, which was uniformly distributed between $-\tau_{\max }$ and $\tau_{\max }$, where $\tau_{\max }$ represents the maximum delay. We assumed that a raisedcosine Nyquist filter characterized by the roll-off factor of $\beta(0 \leq \beta \leq 1)$ was employed at each of the SN and the RNs. Furthermore, in Fig. 7(a) $\tau_{\max }$ was varied from $0.05 T_{\mathrm{S}}$ to $0.3 T_{\mathrm{S}}$ and the roll-off factor was set to $\beta=0.5$, while we considered $\tau_{\max }=0.25 T_{\mathrm{S}}$ as well as $\beta=0,0.25,0.5,0.75$ and 1 in Fig. 7(b), where $T_{\mathrm{S}}$ denotes the symbol duration. Observe in Fig. 7(a) that upon increasing the value of $\tau_{\max }$, the achievable BER of both the schemes was degraded, noting that our cooperative ACSTSK scheme exhibited a reduced sensitivity against IRS errors than its cooperative OSTBC counterpart. This is owning to the ACSTSK scheme's explicit benefit of avoiding simultaneous transmissions from the RNs, hence encountering a reduced number of interferers. It was also found from Fig. 7(b) that the higher roll-off factor $\beta$ resulted in a degraded BER performance, where as expected, the cooperative ACSTSK scheme outperformed the cooperative OSTBC scheme, similarly to Fig. 7(a).

In Fig. 8, we characterized the achievable BER performance of our $\mathcal{L}=4$ PSK-modulated cooperative $\operatorname{DSTSK}(2,2,4)$ scheme, compared to the $\mathcal{L}=4$ PSK-modulated cooperative $\operatorname{CSTSK}(2,2,4)$ scheme. Furthermore, the cooperative DSTSK and CSTSK schemes employed $\mathcal{L}^{\prime}=4$ PSK and $\mathcal{L}^{\prime}=4$ DPSK at the SN, respectively, where both the cooperative schemes exhibited a normalized transmission rate of $R=1.0$ bits/symbol. In order to characterize the effects of the CSI estimation errors associated with coherent detection, we used 


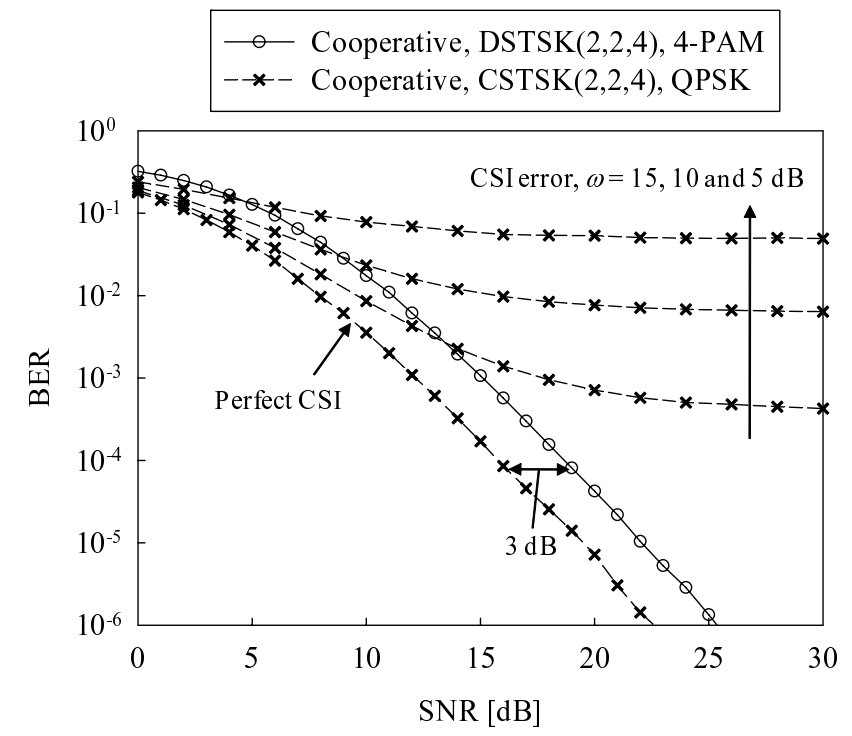

Fig. 8. Achievable BER performance of our $\mathcal{L}=4$ PSK-modulated cooperative $\operatorname{CSTSK}(2,2,4)$ scheme of Fig. 1 and $\mathcal{L}=4$ PSK-modulated cooperative $\operatorname{DSTSK}(2,2,4)$ scheme of Fig. 2, employing $\mathcal{L}^{\prime}=4$ PSK and $\mathcal{L}^{\prime}=4$ DPSK for the SN, respectively. Here, both the cooperative CSTSK and ACSTSK schemes exhibited a normalized transmission rate of $R=1.0 \mathrm{bits} / \mathrm{symbol}$. The effects of the CSI estimation errors associated with the cooperative CSTSK scheme was characterized by an equivalent CSIestimation SNR of $\omega=5,10$ and $15 \mathrm{~dB}$.

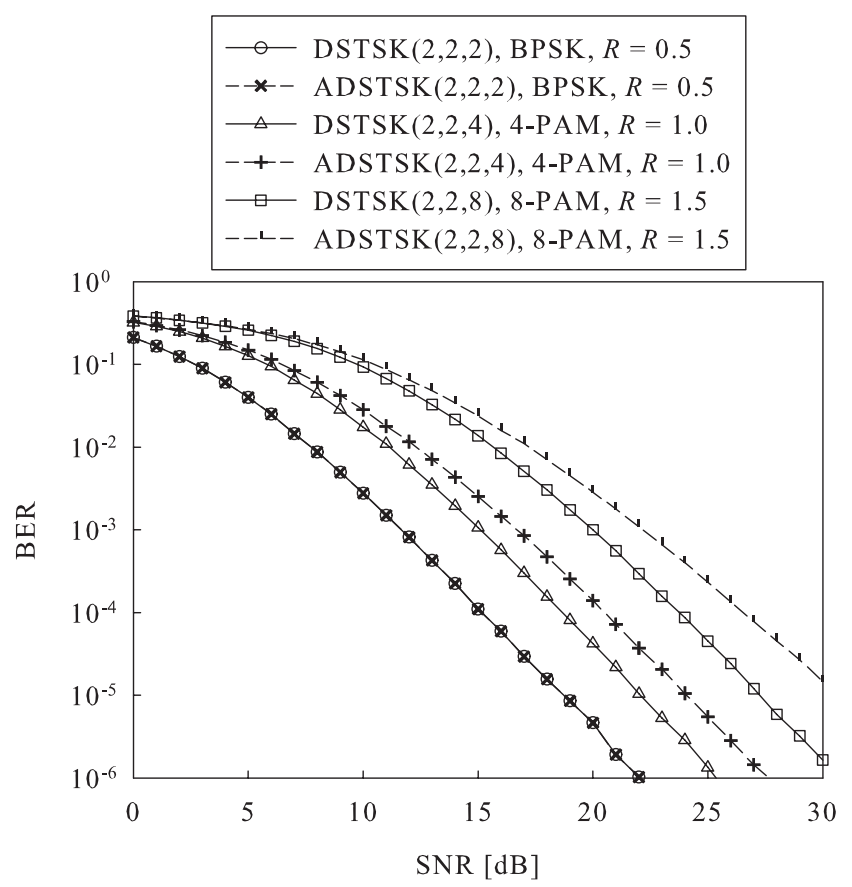

Fig. 9. Comparison of our $\mathcal{L}$-PAM cooperative $\operatorname{DSTSK}(2,2, Q)$ and $\operatorname{ADSTSK}(2,2, Q)$ schemes obeying the architecture of Fig. 2, while employing $\mathcal{L}^{\prime}=\mathcal{L}$ DPSK at the SN. Here, the sets of the parameters of $(\mathcal{L}, Q)$ were given by $(2,2),(4,4)$ and $(8,8)$, achieving the normalized transmission rates of $R=0.5,1.0$ and $1.5 \mathrm{bits} / \mathrm{symbol}$, respectively.

an equivalent CSI-estimation SNR of $\omega=5,10$ and $15 \mathrm{~dB}$. For example, a CSI SNR of $10 \mathrm{~dB}$ indicates that the CSI error power is a factor ten lower than the received signal power. Observe in Fig. 8 that since the equivalent noise variance of the cooperative DSTSK scheme was doubled, the system suffered from a $3 \mathrm{~dB}$ performance penalty in comparison to its coherently-detected counterpart. On the other hand, as expected, the cooperative CSTSK scheme's performance was severely degraded upon introducing the CSI estimation errors, hence exhibiting an error floor. This emphasized the benefits of non-coherent detection at our cooperative DSTSK scheme's receiver.

Finally, we compared the achievable BER performance of our cooperative $\mathcal{L}$-PAM $\operatorname{DSTSK}(2,2, Q)$ and $\operatorname{ADSTSK}(2,2, Q)$ schemes obeying the architecture of Fig. 2, while employing $\mathcal{L}^{\prime}=\mathcal{L}$ DPSK at the SN. Here, the sets of the parameters of $(\mathcal{L}, Q)$ were given by $(2,2),(4,4)$ and $(8,8)$, achieving the normalized transmission rates of $R=0.5,1$ and $1.5 \mathrm{bits} /$ symbol, respectively. It was found in Fig. 9 that while the cooperative DSTSK and ADSTSK schemes having the parameters of $(2,2)$ exhibited a similar BER, a performance difference emerged and became higher upon increasing the values of $(\mathcal{L}, Q)$. This is owing to realvalued diagonal constraint imposed by the cooperative DSTSK scheme.

\section{CONCLUSions}

Motivated by the recent STSK concept, we proposed a novel cooperative CSTSK arrangement, where each RN uses CRC-activated DF relaying employing CSTSK during the cooperative phase of Fig. 1, which is capable of attaining an attractive cooperative diversity gain. Here, the RNs do not require symbol-synchronization owing to the additional restriction which we imposed on the dispersion vector design. At the receiver, the received signals of the direct sourcedestination link and the relay-destination links are jointly detected using IEI-free low-complexity single-stream ML detection. Furthermore, we also proposed a cooperative DSTSK scheme, which dispenses with CSI estimation at all of the nodes, while retaining the benefits of the cooperative CSTSK scheme. More importantly, owing to its design flexibility, our cooperative STSK arrangements enable us to adapt the number of RNs, the transmission rate as well as the achievable diversity order, depending on the associated system requirements and channel conditions.

\section{APPENDIX \\ DiSPERSION-MATRIX SET EMPLOYED FOR OUR Simulations}

Parts of the dispersion-vector sets $\boldsymbol{A}_{q^{\prime}}=$ $\left[\boldsymbol{a}_{q^{\prime}}^{(1) T}, \cdots, \boldsymbol{a}_{q^{\prime}}^{(M) T}\right]^{T} \quad\left(q^{\prime}=1, \cdots, Q\right)$ of $(9)$, which were used for our simulations in this paper are as follows. ${ }^{11}$

- QPSK-modulated cooperative $\operatorname{CSTSK}(2,2,4)$ scheme

${ }^{11}$ Again, since the matrices $\boldsymbol{A}_{q^{\prime}}\left(q^{\prime}=1, \cdots, Q\right)$ of our cooperative $\operatorname{CSTSK}(M, T, Q)$ scheme have the size of $(M \times T)$, their search space increases upon increasing the number of RNs $M$, the number of symbols $T$ per block and the number of dispersion-vector sets $Q$, in addition to the constellation points $\mathcal{L}$. According to the rank- and determinant-criterion, an exhaustive search was implemented in order to optimize $Q$ dispersion-vector sets $\boldsymbol{A}_{q^{\prime}}\left(q^{\prime}=1, \cdots, Q\right)$. As the result, any two of the $Q \cdot \mathcal{L}$ virtual spacetime matrices $s_{l} \boldsymbol{A}_{q}(1 \leq q \leq Q, 1 \leq l \leq \mathcal{L})$ exhibited a low correlation, which offers a good detection performance at the receiver. 


$$
\begin{aligned}
& \boldsymbol{A}_{1}=\left[\begin{array}{rr}
0.0002+j 0.1810 & 0.8053+j 0.0538 \\
-1.0650-j 0.3093 & -0.2929+j 0.0047
\end{array}\right], \\
& \boldsymbol{A}_{2}=\left[\begin{array}{rr}
-0.0945+j 0.9968 & -0.6147+j 0.0826 \\
0.1045-j 0.1268 & -0.7007-j 0.3077
\end{array}\right], \\
& \boldsymbol{A}_{3}=\left[\begin{array}{rr}
-0.8263-j 0.2239 & 0.2992+j 0.6753 \\
0.0804+j 0.0062 & -0.8362+j 0.1261
\end{array}\right], \\
& \boldsymbol{A}_{4}=\left[\begin{array}{rr}
-0.4286-j 0.1218 & -0.4714-j 0.2877 \\
-0.5521-j 0.5868 & -0.0195+j 0.9203
\end{array}\right] .
\end{aligned}
$$

- QPSK-modulated cooperative $\operatorname{ACSTSK}(2,2,4)$ scheme

$$
\begin{aligned}
& \boldsymbol{A}_{1}=\left[\begin{array}{rr}
0.5805+j 1.0840 & 0.0000+j 0.0000 \\
0.0000+j 0.0000 & 0.4475-j 0.5363
\end{array}\right], \\
& \boldsymbol{A}_{2}=\left[\begin{array}{rr}
0.0000+j 0.0000 & 0.9072-j 0.9722 \\
-0.4010-j 0.2666 & 0.0000+j 0.0000
\end{array}\right], \\
& \boldsymbol{A}_{3}=\left[\begin{array}{rr}
0.0000+j 0.0000 & 0.1289-j 0.4211 \\
-0.6341-j 1.1849 & 0.0000+j 0.0000
\end{array}\right], \\
& \boldsymbol{A}_{4}=\left[\begin{array}{rr}
-0.7218-j 0.2186 & 0.0000+j 0.0000 \\
0.0000+j 0.0000 & 0.9082+j 0.7787
\end{array}\right] .
\end{aligned}
$$

- 8-PSK modulated cooperative $\operatorname{CSTSK}(3,2,8)$ scheme

$$
\begin{aligned}
& \boldsymbol{A}_{1}=\left[\begin{array}{rr}
-0.3004-j 0.0851 & -0.5751+j 0.1096 \\
-0.1276+j 1.0183 & -0.2113+j 0.1873 \\
-0.3897+j 0.1733 & -0.3467-j 0.3532
\end{array}\right], \\
& \boldsymbol{A}_{2}=\left[\begin{array}{rr}
0.0417-j 0.1950 & 0.6609+j 0.3697 \\
-0.3282+j 0.6485 & 0.7036-j 0.1895 \\
0.3958+j 0.3428 & -0.1143-j 0.2006
\end{array}\right], \\
& \boldsymbol{A}_{3}=\left[\begin{array}{rr}
0.6896-j 0.2101 & 0.2777+j 0.3861 \\
0.1139+j 0.1366 & 0.3816-j 0.0568 \\
-0.1513+j 0.3383 & 0.5344+j 0.8067
\end{array}\right], \\
& \boldsymbol{A}_{4}=\left[\begin{array}{rr}
-0.4194+j 0.3000 & -0.1861+j 0.0637 \\
-0.4607+j 0.3887 & 0.2187+j 0.6741 \\
-0.0900-j 0.2984 & 0.1206+j 0.8475
\end{array}\right], \\
& \boldsymbol{A}_{5}=\left[\begin{array}{rr}
-0.3674-j 0.0757 & -0.1060+j 0.4035 \\
0.0179-j 0.3796 & 0.2175+j 0.3668 \\
-0.8406+j 0.0350 & -0.1652-j 0.7899
\end{array}\right], \\
& \boldsymbol{A}_{6}=\left[\begin{array}{rr}
-0.3842-j 0.4024 & 0.3809-j 0.2354 \\
-0.3558-j 0.3678 & 0.2873-j 0.6271 \\
-0.2225+j 0.4551 & 0.4703+j 0.5240
\end{array}\right], \\
& \boldsymbol{A}_{8}=\left[\begin{array}{rr}
-0.1888-j 0.5969 & -0.5091-j 0.2274 \\
0.3276+j 0.0346 & -0.3845+j 0.5597 \\
-0.1933+j 0.2262 & 0.3727-j 0.7072 \\
0.4781-j 0.5985 & -0.3340-j 0.2010 \\
-0.4031-j 0.1665 & 0.5244+j 0.1485 \\
0.4878+j 0.4562 & 0.5505+j 0.1582
\end{array}\right],
\end{aligned}
$$

- 8-PSK modulated cooperative $\operatorname{ACSTSK}(3,2,8)$ scheme

$$
\begin{aligned}
& \boldsymbol{A}_{1}=\left[\begin{array}{rr}
0.0000+j 0.0000 & -0.3861-j 0.8623 \\
-0.5232+j 0.9130 & 0.0000+j 0.0000 \\
0.0000+j 0.0000 & 0.0000+j 0.0000
\end{array}\right], \\
& \boldsymbol{A}_{2}=\left[\begin{array}{rr}
-0.6769+j 0.0050 & 0.0000+j 0.0000 \\
0.0000+j 0.0000 & -1.1901+j 0.3544 \\
0.0000+j 0.0000 & 0.0000+j 0.0000
\end{array}\right], \\
& \boldsymbol{A}_{3}=\left[\begin{array}{rr}
0.0000+j 0.0000 & -0.1193+j 0.6103 \\
0.0000+j 0.0000 & 0.0000+j 0.0000 \\
0.2542-j 1.2445 & 0.0000+j 0.0000
\end{array}\right],
\end{aligned}
$$

$$
\begin{aligned}
& \boldsymbol{A}_{4}=\left[\begin{array}{rr}
0.0000+j 0.0000 & 0.0000+j 0.0000 \\
0.0000+j 0.0000 & 0.8381+j 0.3463 \\
0.7953+j 0.7384 & 0.0000+j 0.0000
\end{array}\right], \\
& \boldsymbol{A}_{5}=\left[\begin{array}{rr}
0.0000+j 0.0000 & 0.9194+j 0.6571 \\
0.1888+j 0.8291 & 0.0000+j 0.0000 \\
0.0000+j 0.0000 & 0.0000+j 0.0000
\end{array}\right], \\
& \boldsymbol{A}_{6}=\left[\begin{array}{rr}
-0.2003+j 1.1839 & 0.0000+j 0.0000 \\
0.0000+j 0.0000 & -0.5107+j 0.5454 \\
0.0000+j 0.0000 & 0.0000+j 0.0000
\end{array}\right], \\
& \boldsymbol{A}_{7}=\left[\begin{array}{rr}
0.0000+j 0.0000 & -1.0870+j 0.6871 \\
0.0000+j 0.0000 & 0.0000+j 0.0000 \\
-0.5024-j 0.3063 & 0.0000+j 0.0000
\end{array}\right], \\
& \boldsymbol{A}_{8}=\left[\begin{array}{rr}
0.0000+j 0.0000 & 0.0000+j 0.0000 \\
0.0000+j 0.0000 & -0.8485-j 0.7975 \\
0.6900-j 0.4099 & 0.0000+j 0.0000
\end{array}\right] .
\end{aligned}
$$

Similarly, the dispersion-vector sets of our cooperative $\operatorname{DSTSK}(M, T, Q)$ scheme are give as follows.

- BPSK-modulated

$\operatorname{DSTSK}(2,2,2) / \operatorname{ADSTSK}(2,2,2)$ schemes

cooperative

$$
\begin{aligned}
& \boldsymbol{A}_{1}=\left[\begin{array}{rr}
-2.0355+j 0.0000 & 0.0000+j 0.0000 \\
0.0000+j 0.0000 & 2.0851+j 0.0000
\end{array}\right], \\
& \boldsymbol{A}_{2}=\left[\begin{array}{rr}
-0.4778+j 0.0000 & 0.0000+j 0.0000 \\
0.0000+j 0.0000 & -0.4937+j 0.0000
\end{array}\right] .
\end{aligned}
$$

- 4-PAM cooperative $\operatorname{DSTSK}(2,2,4)$ scheme

$$
\begin{aligned}
& \boldsymbol{A}_{1}=\left[\begin{array}{rr}
-0.1216+j 0.0000 & -0.6216-j 0.2135 \\
-0.6216+j 0.2135 & 0.6667+j 0.0000
\end{array}\right], \\
& \boldsymbol{A}_{2}=\left[\begin{array}{rr}
-1.2083+j 0.0000 & -0.1505+j 0.6034 \\
-0.1505-j 0.6034 & 1.1274+j 0.0000
\end{array}\right], \\
& \boldsymbol{A}_{3}=\left[\begin{array}{rr}
0.0195+j 0.0000 & -0.1032+j 0.9672 \\
-0.1032-j 0.9672 & 0.0264+j 0.0000
\end{array}\right], \\
& \boldsymbol{A}_{4}=\left[\begin{array}{rr}
0.5826+j 0.0000 & -0.5251+j 0.1942 \\
-0.5251-j 0.1942 & -0.5591+j 0.0000
\end{array}\right],
\end{aligned}
$$

- 4-PAM cooperative $\operatorname{ADSTSK}(2,2,4)$ scheme

$$
\begin{aligned}
& \boldsymbol{A}_{1}=\left[\begin{array}{rr}
-0.1826+j 0.0000 & 0.0000+j 0.0000 \\
0.0000+j 0.0000 & -1.3128+j 0.0000
\end{array}\right], \\
& \boldsymbol{A}_{2}=\left[\begin{array}{rr}
-2.5107+j 0.0000 & 0.0000+j 0.0000 \\
0.0000+j 0.0000 & 0.8974+j 0.0000
\end{array}\right], \\
& \boldsymbol{A}_{3}=\left[\begin{array}{rr}
1.0886+j 0.0000 & 0.0000+j 0.0000 \\
0.0000+j 0.0000 & -1.9327+j 0.0000
\end{array}\right], \\
& \boldsymbol{A}_{4}=\left[\begin{array}{rr}
-1.5037+j 0.0000 & 0.0000+j 0.0000 \\
0.0000+j 0.0000 & -0.2140+j 0.0000
\end{array}\right],
\end{aligned}
$$

- 8-PAM cooperative $\operatorname{DSTSK}(2,2,8)$ scheme

$$
\begin{aligned}
& \boldsymbol{A}_{1}=\left[\begin{array}{rr}
-1.4721+j 0.0000 & -0.3984+j 0.3992 \\
-0.3984-j 0.3992 & 0.6660+j 0.0000
\end{array}\right], \\
& \boldsymbol{A}_{2}=\left[\begin{array}{rr}
-0.6168+j 0.0000 & 0.0762+j 0.9948 \\
0.0762-j 0.9948 & -0.2529+j 0.0000
\end{array}\right], \\
& \boldsymbol{A}_{3}=\left[\begin{array}{rr}
0.9270+j 0.0000 & 0.8177+j 0.3899 \\
0.8177-j 0.3899 & -0.7440+j 0.0000
\end{array}\right], \\
& \boldsymbol{A}_{4}=\left[\begin{array}{rr}
0.1543+j 0.0000 & 0.7122-j 0.0597 \\
0.7122+j 0.0597 & -0.3334+j 0.0000
\end{array}\right], \\
& \boldsymbol{A}_{5}=\left[\begin{array}{lr}
0.0126+j 0.0000 & 0.2049+j 0.6086 \\
0.2049-j 0.6086 & -0.6106+j 0.0000
\end{array}\right],
\end{aligned}
$$




$$
\begin{aligned}
& \boldsymbol{A}_{6}=\left[\begin{array}{rr}
-0.6332+j 0.0000 & 0.0462+j 0.3991 \\
0.0462-j 0.3991 & 0.1955+j 0.0000
\end{array}\right] \\
& \boldsymbol{A}_{7}=\left[\begin{array}{rr}
-0.2510+j 0.0000 & 0.7211-j 0.5317 \\
0.7211+j 0.5317 & 0.9348+j 0.0000
\end{array}\right] \\
& \boldsymbol{A}_{8}=\left[\begin{array}{rr}
-0.7305+j 0.0000 & 0.6227+j 0.3666 \\
0.6227-j 0.3666 & 0.1653+j 0.0000
\end{array}\right]
\end{aligned}
$$

- 8-PAM cooperative $\operatorname{ADSTSK}(2,2,8)$ scheme

$$
\begin{aligned}
& \boldsymbol{A}_{1}=\left[\begin{array}{rr}
-0.6704+j 0.0000 & 0.0000+j 0.0000 \\
0.0000+j 0.0000 & -1.2194+j 0.0000
\end{array}\right], \\
& \boldsymbol{A}_{2}=\left[\begin{array}{rr}
2.0942+j 0.0000 & 0.0000+j 0.0000 \\
0.0000+j 0.0000 & -0.3265+j 0.0000
\end{array}\right], \\
& \boldsymbol{A}_{3}=\left[\begin{array}{rr}
1.0534+j 0.0000 & 0.0000+j 0.0000 \\
0.0000+j 0.0000 & 0.6948+j 0.0000
\end{array}\right], \\
& \boldsymbol{A}_{4}=\left[\begin{array}{lr}
0.1616+j 0.0000 & 0.0000+j 0.0000 \\
0.0000+j 0.0000 & 1.5990+j 0.0000
\end{array}\right], \\
& \boldsymbol{A}_{5}=\left[\begin{array}{rr}
0.2940+j 0.0000 & 0.0000+j 0.0000 \\
0.0000+j 0.0000 & -1.3817+j 0.0000
\end{array}\right], \\
& \boldsymbol{A}_{6}=\left[\begin{array}{rr}
0.8357+j 0.0000 & 0.0000+j 0.0000 \\
0.0000+j 0.0000 & 0.3733+j 0.0000
\end{array}\right], \\
& \boldsymbol{A}_{7}=\left[\begin{array}{rr}
1.4136+j 0.0000 & 0.0000+j 0.0000 \\
0.0000+j 0.0000 & -2.1170+j 0.0000
\end{array}\right], \\
& \boldsymbol{A}_{8}=\left[\begin{array}{rr}
-0.5276+j 0.0000 & 0.0000+j 0.0000 \\
0.0000+j 0.0000 & 0.1621+j 0.0000
\end{array}\right] .
\end{aligned}
$$

\section{REFERENCES}

[1] J. Laneman and G. Wornell, "Distributed space-time-coded protocols for exploiting cooperative diversity in wireless networks," IEEE Trans. Inf. Theory, vol. 49, no. 10, pp. 2415-2425, 2003.

[2] R. Nabar, H. Bolcskei, and F. Kneubuhler, "Fading relay channels: performance limits and space-time signal design," IEEE J. Sel. Areas Commun., vol. 22, no. 6, pp. 1099-1109, 2004.

[3] T. Wang, A. Cano, G. Giannakis, and J. Laneman, "High-performance cooperative demodulation with decode-and-forward relays," IEEE Trans. Commun., vol. 55, no. 7, pp. 1427-1438, 2007.

[4] R. C. Palat, A. Annamalai, and J. H. Reed, "Accurate bit-error-rate analysis of bandlimited cooperative OSTBC networks under timing synchronization errors," IEEE Trans. Veh. Technol., vol. 58, no. 5, pp. 2191-2200, 2009.

[5] X. Li, "Space-time coded multi-transmission among distributed transmitters without perfect synchronization," IEEE Signal Process. Lett., vol. 11, no. 12, pp. 948-951, 2004.

[6] M. Sharp, A. Scaglione, and B. Sirkeci-Mergen, "Randomized cooperation in asynchronous dispersive links," IEEE Trans. Commun., vol. 57, no. 1, pp. 64-68, 2009.

[7] S. Sugiura, S. Chen, and L. Hanzo, "Cooperative differential spacetime spreading for the asynchronous relay aided CDMA uplink using interference rejection spreading code," IEEE Signal Process. Lett., vol. 17 , no. 2, pp. 117-120, 2010.

[8] P. Tarasak, H. Minn, and V. Bhargava, "Differential modulation for two-user cooperative diversity systems," IEEE J. Sel. Areas Commun., vol. 23, no. 9, pp. 1891-1900, 2005.

[9] T. Himsoon, W. Su, and K. Liu, "Differential transmission for amplifyand-forward cooperative communications," IEEE Signal Process. Lett., vol. 12, no. 9, pp. 597-600, Sep. 2005.

[10] R. Mesleh, H. Haas, S. Sinanovic, C. Ahn, and S. Yun, "Spatial modulation," IEEE Trans. Veh. Technol., vol. 57, no. 4, pp. 2228-2242, 2008.

[11] J. Jeganathan, A. Ghrayeb, and L. Szczecinski, "Spatial modulation: optimal detection and performance analysis," IEEE Commun. Lett., vol. 12 , no. 8 , pp. $545-547,2008$.

[12] J. Jeganathan, A. Ghrayeb, L. Szczecinski, and A. Ceron, "Space shift keying modulation for MIMO channels," IEEE Trans. Wireless Commun., vol. 8, no. 7, pp. 3692-3703, 2009.

[13] S. Sugiura, S. Chen, and L. Hanzo, "Coherent and differential spacetime shift keying: a dispersion matrix approach," IEEE Trans. Commun., vol. 58 , no. 11 , pp. $3219-3230,2010$.
[14] — - "Generalized space-time shift keying designed for flexible diversity-, multiplexing- and complexity-tradeoffs," IEEE Trans. Wireless Commun., vol. 10, pp. 1-10, 2011, in press (early access available at IEEE Xplore).

[15] S. Alamouti, "A simple transmit diversity technique for wireless communications," IEEE J. Sel. Areas Commun., vol. 16, no. 8, pp. 1451-1458, 1998.

[16] V. Tarokh, H. Jafarkhani, and A. Calderbank, "Space-time block coding for wireless communications: performance results," IEEE J. Sel. Areas Commun., vol. 17, no. 3, pp. 451-460, 1999.

[17] R. W. Heath, Jr. and A. J. Paulraj, "Linear dispersion codes for MIMO systems based on frame theory," IEEE Trans. Signal Process., vol. 50, no. 10, pp. 2429-2441, 2002.

[18] J. Laneman, D. Tse, and G. Wornell, "Cooperative diversity in wireless networks: efficient protocols and outage behavior," IEEE Trans. Inf. Theory, vol. 50, no. 12, pp. 3062-3080, 2004.

[19] C. Xu, S. Sugiura, S. X. Ng, and L. Hanzo, "Reduced-complexity noncoherently detected differential space-time shift keying," in Proc. IEEE WCNC, Mar. 2011, accepted for publication.

[20] B. Hassibi and B. Hochwald, "Cayley differential unitary space-time codes," IEEE Trans. Inf. Theory, vol. 48, no. 6, pp. 1485-1503, 2002.

[21] _ , "High-rate codes that are linear in space and time," IEEE Trans. Inf. Theory, vol. 48, no. 7, pp. 1804-1824, 2002.

[22] L. Hanzo, O. Alamri, M. El-Hajjar, and N. Wu, Near-Capacity MultiFunctional MIMO Systems: Sphere-Packing, Iterative Detection and Cooperation. John Wiley and IEEE Press, 2009.

[23] J. Wang, X. Wang, and M. Madihian, "On the optimum design of spacetime linear-dispersion codes," IEEE Trans. Wireless Commun., vol. 4, no. 6, pp. 2928-2938, 2005.

[24] V. Tarokh, S. Alamouti, and P. Poon, "New detection schemes for transmit diversity with no channel estimation," in Proc. International Conf. Universal Pers. Commun., 1998, vol. 2, pp. 917-920.

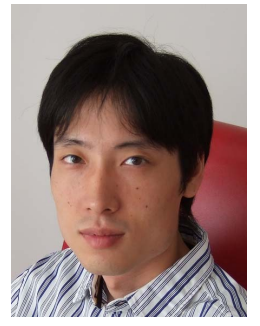

Shinya Sugiura (M'06) received the B.S. and M.S. degrees from Kyoto University, Kyoto, Japan, in 2002 and 2004, respectively, and the Ph.D. degree from the University of Southampton, Southampton, UK, in 2010. Since 2004, he has been with Toyota Central R\&D Laboratories, Inc., Japan. His research has covered a range of areas in communications, including space-time modulation/demodulation, turbo coding, cooperative communications, multiuser detection, and automotive antenna design, as well as vehicular ad hoc networking.

Dr. Sugiura has published more than 16 journal papers and 17 refereed conference papers. He was awarded the IEEE AP-S Japan Chapter Young Engineer Award in December 2008.

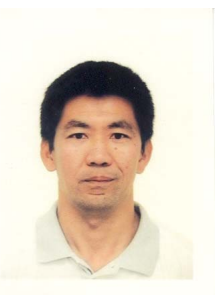

Sheng Chen (M'90-SM'97-F'08) obtained a BEng degree from the East China Petroleum Institute, Dongying, China, in 1982, and a $\mathrm{PhD}$ degree from the City University, London, in 1986, both in control engineering. In 2005, he was awarded the DSc from the University of Southampton, Southampton, UK. Since 1999, he has been with the School of Electronics and Computer Science, the University of Southampton, UK. He previously held research and academic appointments at the Universities of Sheffield, Edinburgh, and Portsmouth, all in the UK. Professor Chen's recent research works include adaptive signal processing, wireless communications, modelling and identification of nonlinear systems, neural network and machine learning, finite-precision digital controller design, evolutionary computation methods, and optimization. He has published over 280 research papers. In the database of the world's most highly cited researchers in various disciplines, compiled by the Institute for Scientific Information (ISI) of the USA, Prof. Chen is on the list of the highly cited researchers in the engineering category. See http://www.ISIHighlyCited.com 


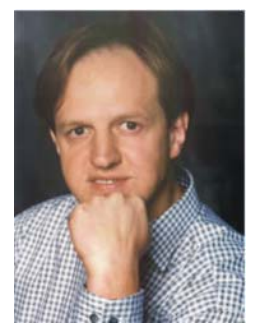

Harald Haas (S'98-AM'00-M'03) received the $\mathrm{Ph} . \mathrm{D}$. degree from the University of Edinburgh in 2001. From 2001 to 2002, he was a research project manager at Siemens in Munich. He joined Jacobs University, Bremen in 2002 as an Associate Professor, before returning in 2007 to the University of Edinburgh, where he now holds a personal Chair in Mobile Communications.

His research interests are decentralised interference management in wireless networks, the spatial modulation multiple antenna concept, and optical

wireless communication.

He holds more than 15 patents in the area of wireless communications, and has published more than 170 conference and journal papers. He co-authors a book entitled Next Generation Mobile Access Technologies: Implementing $T D D$ with Cambridge University Press.

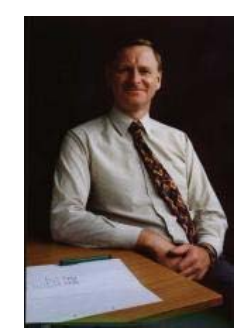

Peter M. Grant (M'77-SM'83-F'96) was born in St. Andrews, Scotland. He received a B.Sc. in 1966 from the Heriot-Watt University, Edinburgh, the $\mathrm{Ph} . \mathrm{D}$. degree from the University of Edinburgh in 1975, and honorary DEng's from the Heriot-Watt in 2006 and from Napier University in 2007.

He was appointed to the University of Edinburgh in 1971, was subsequently promoted to a full professor of electronic signal processing, and then departmental chair from 1999-2002, and head of the School of Engineering at Edinburgh from 2002-

2008.

During the academic year 1977-1978, he was a visiting professor at the Ginzton Laboratory, Stanford University, and in 1985-1986 he was a visiting staff member at the MIT Lincoln Laboratory. He was awarded the 82nd (2004) Faraday Medal by the Institution of Electrical Engineers (IEE).

He was president of EURASIP from 2000-2002, chairman of EUSIPCO94, and technical program chairman for ICASSP-89. From 1998-1999, he was appointed by the IEEE Signal Processing Society as a distinguished lecturer on DSP for Mobile Communications. He served as a director of the Mobile VCE from 2002-2004 and 2007-2009.

He was, in 2007, appointed the 8th Regius Professor of Engineering at The University of Edinburgh. In 2009, he was made an officer of the order of the British Empire (OBE) on the Queen's birthday honours list. He was elected one of the first four EURASIP Fellows and also holds fellowships of the IET, Royal Academy of Engineering, and the Royal Society of Edinburgh.

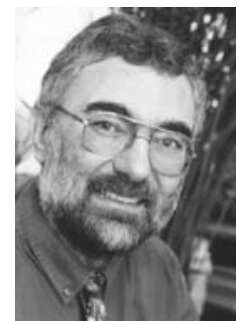

Lajos Hanzo (M'91-SM'92-F'04) FREng, FIEEE, FIET, DSc, received his degree in electronics in 1976 and his doctorate in 1983. During his 34-year career in telecommunications, he has held various research and academic posts in Hungary, Germany, and the UK. Since 1986, he has been with the School of Electronics and Computer Science, University of Southampton, UK, where he holds the chair in telecommunications. He has co-authored 20 John Wiley - IEEE Press books on mobile radio communications totalling in excess of 10,000 pages, published 880 research papers and book chapters on IEEE Xplore, acted as TPC Chair of IEEE conferences, presented keynote lectures, and been awarded a number of distinctions. Currently, he is directing an academic research team, working on a range of research projects in the field of wireless multimedia communications sponsored by industry, the Engineering and Physical Sciences Research Council (EPSRC) UK, the European IST Programme, and the Mobile Virtual Centre of Excellence (VCE), UK. He is an enthusiastic supporter of industrial and academic liaison and he offers a range of industrial courses. He is also an IEEE Distinguished Lecturer as well as a Governor of both IEEE ComSoc and the VTS. He is the Editor-in-Chief of the IEEE Press and a Chaired Professor at Tsinghua University, Beijing. For further information on research in progress and associated publications, please refer to http://www-mobile.ecs.soton.ac.uk 